\title{
Spatial organization of chromosomes leads to heterogeneous chromatin motion and drives the liquid- or gel-like behavior of chromatin
}

\author{
Hossein Salari ${ }^{1,{ }^{*}}$, Marco Di Stefano ${ }^{2,+}$, Daniel Jost ${ }^{1, *}$ \\ ${ }^{1}$ Université de Lyon, ENS de Lyon, Univ Claude Bernard, CNRS, Laboratoire de Biologie et \\ Modélisation de la Cellule, Lyon, France \\ ${ }^{2}$ CNAG-CRG, The Barcelona Institute of Science and Technology (BIST), Barcelona, Spain \\ ${ }^{+}$Present address: Institute of Human Genetics, Univ of Montpellier, CNRS, Laboratoire de \\ Chromatine et Biologie Cellulaire, Montpellier, France. \\ *Corresponding authors: hossein.salari@ens-lyon.fr (H.S.) and daniel.jost@ens-lyon.fr (D.J.)
}

\begin{abstract}
Chromosome organization and dynamics are involved in the regulation of many fundamental processes such as gene transcription and DNA repair. Experiments unveiled that inside cell nuclei chromatin motion is highly heterogeneous ranging from a liquid-like, mobile state to a gel-like, rigid state. Using polymer modeling, we investigated how these different physical states and dynamical heterogeneities may emerge from the same structural mechanisms. We found that the formation of topologically-associating domains (TADs) is a key driver of chromatin motion heterogeneity. In particular, we demonstrated that the local degree of compaction of the TAD regulates the transition from a weakly compact, fluid state of chromatin to a more compact, gel state exhibiting anomalous diffusion and coherent motion. Our work provides a comprehensive study of chromosome dynamics and offers a unified view of chromatin motion allowing to interpret the wide variety of dynamical behaviors observed experimentally across different biological conditions.
\end{abstract}

\section{Introduction}

The structural and dynamical properties of the eukaryotic genome inside cell nucleus play crucial roles in many cell functions, such as gene regulation (1). Over the last decade, the high-throughput chromosome conformation capture $(\mathrm{Hi}-\mathrm{C})$ method has provided valuable information about how genomes organize by measuring the contact frequencies between all pairs of chromatin loci (2). Analyses of $\mathrm{Hi}-\mathrm{C}$ experiments in various species and cell types revealed that interphase chromosomes are partitioned at different scales (3): from topologically-associating domains (TADs) (4) at few hundreds of kilo-basepairs (kbp) to the euchromatic ' $A$ ' and heterochromatic ' $B$ ' compartments at Mbp scales and to chromosome territories at the nuclear scale. Complementary to $\mathrm{Hi}-\mathrm{C}$, advances in microscopy on fixed cells 
confirmed the physical existence of these architectural motifs at the single-cell level (5-8) and showed that at the sub-TAD scale, chromatin organizes into clutches of nucleosomes $(9,10)$ clustered into nanodomains (11).

Beyond the 'static' picture of a layered spatial organization that contributes to genome regulation, more and more experiments on living cells highlighted the 'dynamic' nature of chromatin folding and its importance on key biological functions (12-14). Chromatin mobility has been proposed to impact the dynamics of promoter-enhancer interactions and thus regulate transcriptional bursting $(15,16)$, facilitate homology search after DNA damage (17) or participate in the long-range spreading of epigenomic marks (18). Chromatin motion is standardly investigated by measuring the mean-squared displacement (MSD) after a time-lag $\Delta t$. Many live-tracking experiments (19-32) have shown that the MSD of an individual locus can be interpreted by a power-law $M S D=D \Delta t^{\alpha}$, where $D$ is the diffusion constant and $\alpha$ is the diffusion exponent. A wide variety of diffusion constants and exponents have been observed experimentally (Fig. 1A) depending on the cell type (27), the transcriptional or physiological state of the cell $(21,28,29)$ or the presence of DNA damage $(17,33-35)$. Strikingly in many situations, the MSD may exhibit different diffusion regimes (i.e. different $\alpha$ values) at different time-lag scales. In addition to such heterogeneity across conditions, chromatin motion is also highly heterogeneous inside individual nuclei (Fig.1B,C) as observed by genome-wide experiments of chromatin dynamics $(20,23,27)$ which detected, at a same time, populations of loci with high or low mobility $(20,23,36)$. These studies also revealed the presence of spatial chromatin domains of correlated motions $(24,37)$. However, the determinants driving the mobility of individual loci or the formation of such domains are still unclear as some studies associate them with the hetero/euchromatin compartmentalization $(19,36)$ or cohesinmediated TAD organization (20) while others did not observe significant correlation between such heterogeneity and chromatin compaction (23).
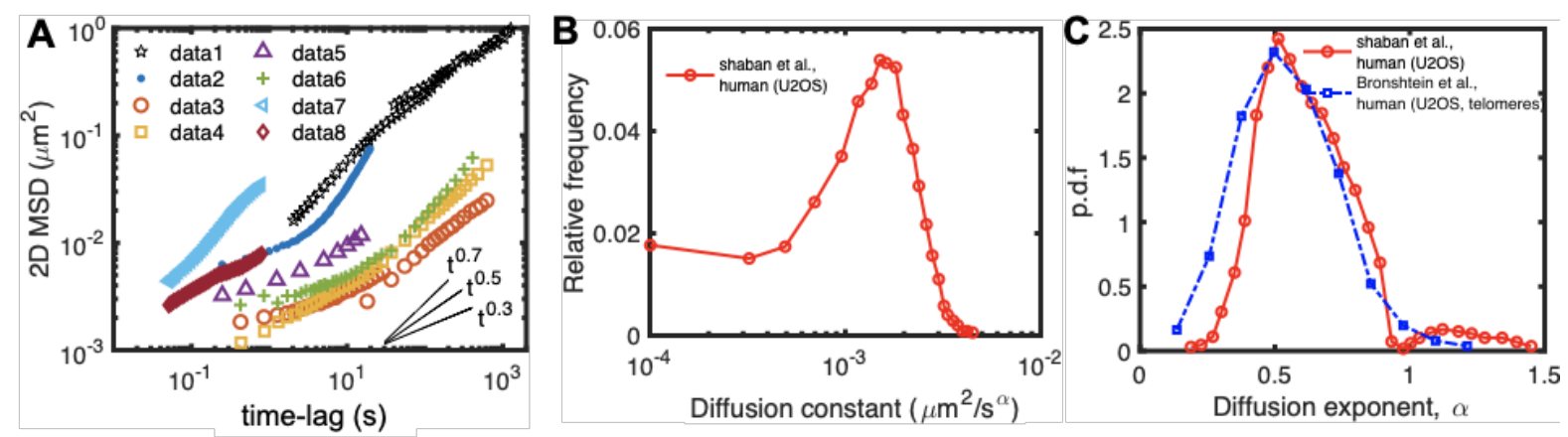

Fig 1: Heterogeneity of chromatin motion. (A) Examples of mean-squared displacement (MSD) profiles measured experimentally at individual loci for different organisms and cell lines. The different datasets are from: (data1) Mouse pro-B (22), (data2) human MCF-7 (not transcribed gene) (21), (data3) human U2OS (centromeres) (27), (data4) human U2OS (telomeres) (27), (data5) human HeLa cells (24), (data6) mouse MF (27), (data7 and data8) human HeLaS3 (fast loci and slow loci, respectively) (20). (B,C) Distributions of diffusion constants (B) and diffusion exponents (C) measured for human U2OS cells (data extracted from $(23,27)$ ).

Biophysical modeling has been instrumental in interpreting and predicting the outcomes of live imaging experiments on chromatin motion $(12,38)$. Indeed, in classical kinetic theory, the value of the diffusion exponent $\alpha$ may be a good indicator of the main underlying physical processes driving the motion of the object under study. For small particles, while standard diffusion is 
characterized by $\alpha=1$, subdiffusion $(\alpha<1)$ and superdiffusion $(\alpha>1)$ may indicate constrained or facilitated movement, respectively. For polymers which are large molecules with many internal degrees of freedom, the fixed connectivity along the chain constrains the motion of individual monomers. The Rouse model, a standard polymer theory assuming that mobility is only driven by thermal fluctuations (39), thus predicts that the loci of the polymer chromatin should experience a subdiffusive motion with $\alpha \sim 0.5$, which is the average typical exponent measured experimentally (Fig.1). One can then define a sub-Rousean $(\alpha<0.5)$ and a super-Rousean $(\alpha>0.5)$ diffusion regimes for polymers that may translate additional constraints or forces on the monomer motion. Therefore, several decorated Rouse-like models have been developed along the years to suggest that the observed sub-Rousean dynamics may be associated with condensation of chromatin $(40,41)$ and the super-Rousean regimes with active processes $(42,43)$ (see $(12)$ for a review). Dynamical simulations of copolymer models capturing quantitatively the different layers of chromosome organization $(40,41,44-47)$ are consistent with an average sub-Rousean regime, with different mobilities between eu- and heterochromatic regions and with correlated motion associated with compartmentalization. In particular, Shi et al associated the experimentally-observed heterogeneity in chromatin motion to the intrinsic glassy dynamics of chromosomes (40), while Shukron et al suggested that it emerges from cell-to-cell variability in cross-linking sites (46)(47).

All these experimental and theoretical works draw a composite - and relatively controversial picture of how chromatin moves inside cell nuclei during interphase and of how this heterogeneity in motion emerges from fundamental processes and from chromatin architecture. In particular, this has lead to two main descriptions of chromatin motion, based on an analogy with material science (48): chromatin behaves like a 'liquid' or a 'fluid' $(49,50)$ pointing to a dynamic and mobile view of chromatin motion; or behaves like a 'gel' or a 'solid' $(22,51)$ highlighting a more constrained and rigid dynamics.

In order to shed light into this controversy, we investigated how the heterogeneous and anomalous behaviors of chromatin mobility may emerge from first principles. In particular, we addressed the interplay between the three-dimensional chromosome organization and the different diffusion regimes of chromatin observed experimentally by investigating the dynamics of heteropolymer models that quantitatively describe the chromosome architecture. We showed that heterogeneity in the local chromatin dynamics is tightly linked to genome folding. Our findings suggest that the TAD layer of organization is the main driver of this dynamical heterogeneity: loci inside weakly compact TADs exhibit a fluid-like behavior with standard, Rouse-like diffusion regimes, while loci inside compact TADs exhibit anomalous diffusion and transition between sub- and super-Rousean regimes, signature of a local gel-like state with coherent motion. Our work offers a unified view of chromatin motion and leverages many apparent paradoxes coming from the different studies of chromosome dynamics suggesting that the 'liquid' or 'solid' behaviour of chromatin are in fact two sides of the same coin. 

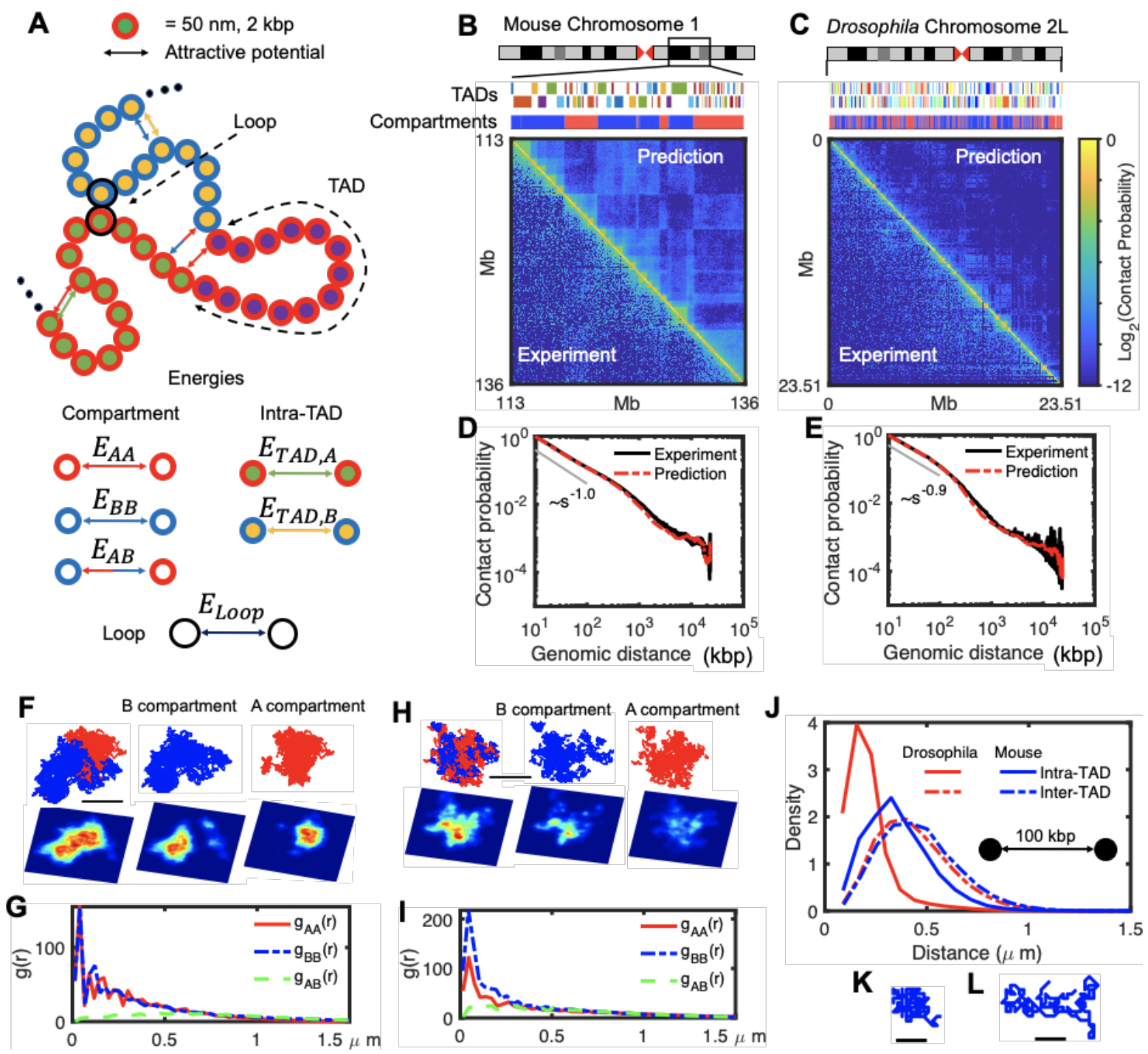

Fig 2: The heteropolymer model and comparison with Hi-C maps. (A) Schematic representation of Heteropolymer model with different structural components and their associated interactions. Each monomer hosts $2 \mathrm{kbp}$ of DNA and its diameter is $50 \mathrm{~nm}$. The surrounding red (blue) color indicates A (B) compartment, inner different colors correspond to different TADs, and loop anchors are shown by black outer circles. All six possible attractive interactions are shown by arrows in this cartoon. (B,C) Visual comparison of predicted $\mathrm{Hi}-\mathrm{C}$ maps and the experimental ones for $23 \mathrm{Mbp}$ of mouse chromosome 1 (113-136 Mbp) and Drosophila chromosome 2L. The upper tracks show the corresponding TADs and compartments for each chromosome. (D,E) Contact probabilities extracted from predicted (red) and experimental (black) Hi-C maps shown in panels (B) and (C), respectively. $(\mathbf{F}, \mathrm{H})$ Typical snapshots for mouse chr1 $(\mathrm{F})$ and Drosophila chr2L $(\mathrm{H})$ in mixed and separate A (red) and $\mathrm{B}$ (blue) compartments, with corresponding 2D density plots (the lower panels) with axial resolution of $1200 \mathrm{~nm}$, lateral resolution of $100 \mathrm{~nm}$ and pixel size of $50 \mathrm{~nm}$. Bars represent $1 \mu \mathrm{mof}$ real space. (G,I) Radial distribution functions $(g$ ) between A-A (red), B-B (blue) and A-B (green) monomers for mouse (G) and Drosophila (I) . (J) Comparison between intra- (full curves) and inter-TAD (dashed curves) distance distributions of pair monomers separated by $100 \mathrm{kbp}$ along the genome for Drosophila (red) and mouse (blue). The typical snapshots of $\sim 300 \mathrm{kbp}$ long TADs for Drosophila (K) and mouse (L). Bars represents $0.25 \mu m$. 


\section{Results}

\section{Quantitative modeling of 3D chromosome organization}

To investigate chromatin motion in situations compatible with experiments, we first developed a polymer model to quantitatively describe the 3D chromatin organization. We modeled chromatin as a coarse-grained heteropolymer (Fig. 2A). Each monomer, containing $2 \mathrm{kbp}$ of DNA and being of size $50 \mathrm{~nm}$, is characterized by three structural features inferred from $\mathrm{Hi}-\mathrm{C}$ maps (see Methods): the TAD it belongs to, its related compartment ( $A$ or $B$ ), and if it corresponds to the anchor of a CTCF-mediated loop as often observed between TAD boundaries in mammals $(52,53)$. The spatio-temporal dynamics of the system is governed by generic properties of a homopolymer (excluded volume and bending rigidity) (45) decorated by three types of short-ranged attractive interactions accounting for the heterogeneity of monomer states (see Fig.2A and Methods): intra- and inter-compartment ( $E_{A A}, E_{B B}, E_{A B}$ ), intraTAD with a strength that depends on the local compartmentalization $\left(E_{T A D, A}, E_{T A D, B}\right)$, and looping between CTCF anchors $\left(E_{\text {loop }}\right)$.

Next, we optimized the parameters (Methods) of the model for two regions of interest (Table 1): a $23 \mathrm{Mbp}$-long portion of chromosome 1 (113-136 Mbp) in mouse embryonic stem cells (mESC) (Fig.2B) and chromosome arm 2L in Drosophila melanogaster Kc167 female embryonic cells (Fig.2C). Note that our choice for these two cell types was motivated by the possibility to investigate two different situations with different TAD and compartment sizes (see the upper tracks in Fig.2B,C) and compaction levels but under the same first principles modeling framework.

Briefly, we used Hi-C maps as an input to detect TADs, compartments and loop anchors, and thus to define the state of each monomer. Then, by varying the energy interactions, we inferred for each species the parameter set that best predicts the experimental Hi-C (see Methods and Table 2). In both cases, the heteropolymer model was able to quantitatively reproduce the $\mathrm{Hi}-\mathrm{C}$ data with an overall very high accuracy (correlation of 0.95 with mouse data and 0.87 for Drosophila) (Fig.2B-C). This goodness of fit, not only captures the average generic decay of contact frequency as a function of their genomic distance (Fig.2D-E), but also the structural features of Hi-C maps at different scales (see Suppl. Fig. S1).

At large scales, the checkerboard-like patterns observed in $\mathrm{Hi}-\mathrm{C}$ maps suggest that chromatin compartments are spatially segregated. Typical configuration snapshots from our simulations in the drosophila (Fig.2F) and in the mouse (Fig.2G) examples indeed illustrate the relative organization of $\mathrm{A}$ and $\mathrm{B}$ compartments. We quantified this by computing the radial distribution functions (Fig.2H-I) $g_{A A}(r), g_{B B}(r)$ and $g_{A B}(r)$ that capture the probabilities to find a monomer of a given compartment at a given distance $r$ from a monomer of the same $\left(g_{A A}(r), g_{B B}(r)\right)$ or of a different compartment $\left(g_{A B}(r)\right)$. In Drosophila, we observed that the B compartment is locally more compact than $\mathrm{A}\left(g_{B B}(r)>g_{A A}(r)\right.$ for small $r$, Fig.2H), while compaction is similar in both compartments in mouse (Fig.2I). Interestingly, we remarked that the $A$ and $B$ compartments are more segregated in mouse with $g_{A B}(r)$ equating $g_{A A / B B}(r)$ around $r \simeq 700$ $\mathrm{nm}$ (vs r $\simeq 300 \mathrm{~nm}$ in Drosophila), resulting in part from the larger genomic size of compartments in the mouse case (see upper tracks Fig.2B vs Fig.2C). 
At the TAD-scale, structural properties are also strongly cell type- and compartmentdependent. On average, TADs in mice (median size $\sim 120 \mathrm{kbp}$ ) are larger than in drosophila (median size 40 kbp) (Fig 2B and 2C upper tracks). Globally, TADs in the drosophila case are more compact than in mice (two typical snapshots of TADs with same size are drawn in Fig.2K-L) with a relatively smaller ratio of intra- versus inter-TAD distances of drosophila compared to mice (Fig.2J). Similar to the condensation of compartments (Fig.2H-I), we also observed that TADs in the A compartment are less compacted than those in B for drosophila $(7,54)(55)$, while being more open and having similar compaction level in the mouse case (56) (Suppl. Fig. S1G,H).
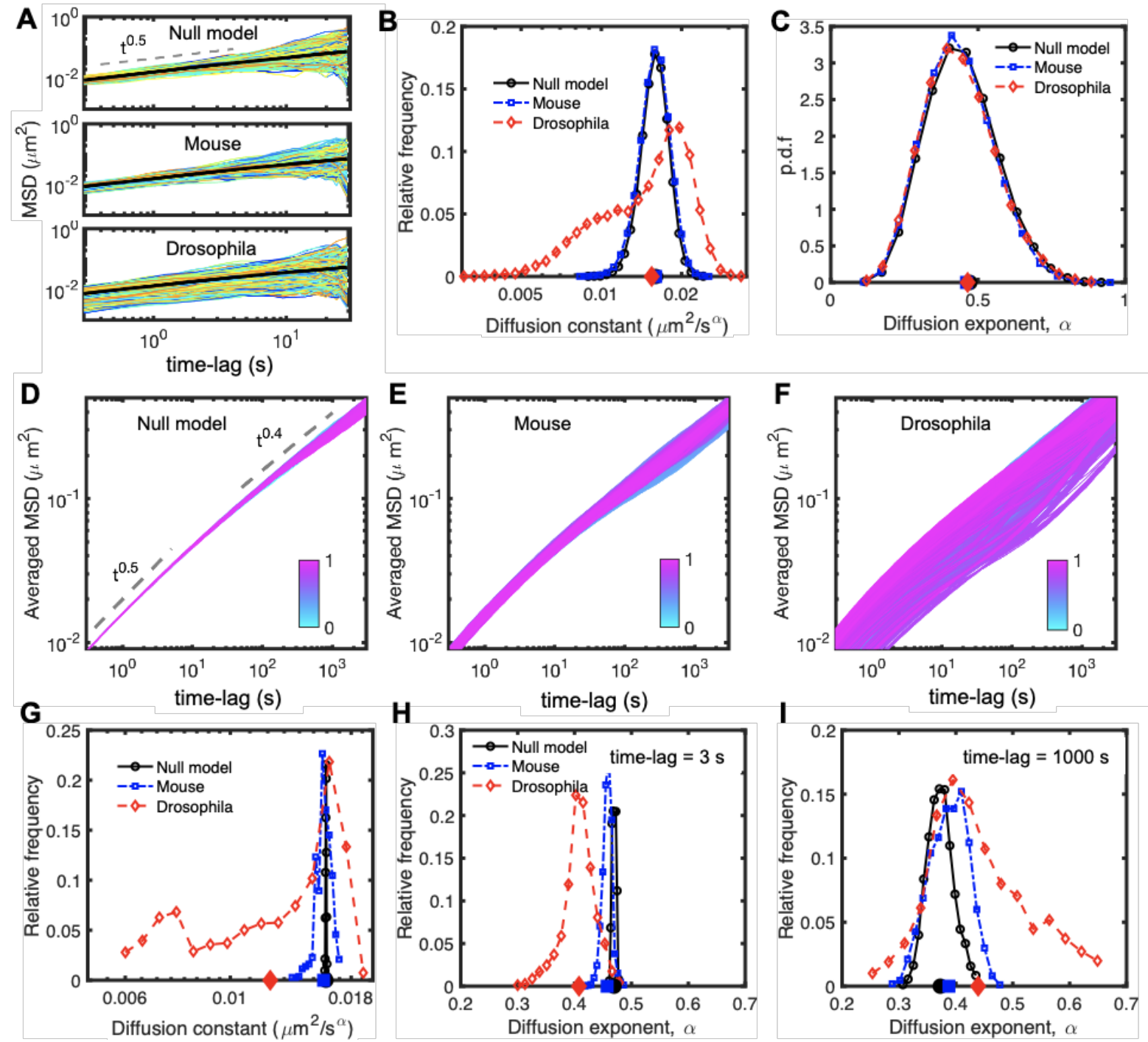

Fig 3: Dynamic properties of simulated chromosomes. (A) Time averaged MSD of single trajectories of $30 \mathrm{~s}$ sampled every $0.3 \mathrm{~s}$ for null (upper panel), mouse (middle panel) and Drosophila (bottom panel) models. (B,C) The distributions of the diffusion constant $D_{i, c}(\mathrm{~B})$ and exponent $\alpha_{i, c}(\mathrm{C})$ of trajectories in panel (A). The average values are shown on the horizontal axis. (D,E,F) Ensembleaveraged (over all trajectories) $M S D_{i}$ of all genomic loci for null $(\mathrm{D})$, mouse $(\mathrm{E})$ and Drosophila $(\mathrm{F})$ models. To discard trivial positional effects, we exclude the last $100 \mathrm{kbp}$ loci at the two ends of the polymers. Individual MSDs were colored from cyan (first monomer) to magenta (last monomer). (G,H,I) The distributions of the diffusion constant $D_{i}$ at very short time scale $(\mathrm{G})$ and of the diffusion exponent 
$\alpha_{i}$ for short $(\mathrm{H})$ and large (I) time scales, extracted from MSD curves in panels (D-F). The average values are shown on the horizontal axis.

\section{Chromatin dynamics is strongly heterogeneous and locus- dependent}

Having in hand a quantitative polymer model predictive of the main structural features of chromosome organization, we investigated the dynamical properties of chromatin. As a reference, null model, we also simulated the dynamics of a simple homopolymer where all compartment-, TADs-, or loop-based interactions were switched off (see Methods). One standard observable to probe the local chromatin mobility is the mean-squared displacement (MSD) of individual loci as a function of time. Experimentally, this is typically done either by tracking single fluorescently-labelled loci (32) or by monitoring the dynamics of the nuclear local densities of stained histones (24) during 10-30 seconds. For each single trajectory extracted from these experiments, time-averaged MSDs can be estimated with $\operatorname{MSD}_{i, c}(\Delta t) \equiv$ $\left\langle\left(\vec{r}_{i, c}(t+\Delta t)-\vec{r}_{i, c}(t)\right)^{2}\right\rangle_{t}$ where $\vec{r}_{i, c}(t)$ is the position vector of a given locus $i$ at time $t$ for trajectory $c$ and $\langle\cdots\rangle_{t}$ is the time-averaging along $c$. By fitting them with a power-law $M S D_{i, c}(\Delta t)=D_{i, c} \Delta t^{\alpha_{i, c}}$, both experimental methods have shown high variability in the diffusion constant $D_{i, c}$ and exponent $\alpha_{i, c}$ at the single-cell level in many species and for many loci (see examples in Fig.1B,C) $(19,20,23,24)$. By ensemble-averaging over many trajectories $\left(M S D_{i}(\Delta t) \equiv\left\langle\left(\vec{r}_{i, c}(t+\Delta t)-\vec{r}_{i, c}(t)\right)^{2}\right\rangle_{t, c}=D_{i} \Delta t^{\alpha_{i}}\right)$, single locus tracking experiments exhibit a wide variety of sub-diffusive $\alpha_{i}$-values depending on the locus, species or transcriptional state $(21,27,28,30,31)$, with sometimes crossovers between different regimes (see some examples in Fig. 1A).

To try to understand the origin of this heterogeneity, we first estimated with our simulations the time-averaged MSD for all the monomers in the Drosophila and mouse cases as well as for the null model over 30 seconds-long trajectories (Fig.3A). Interestingly, our models predicted a large variability of diffusion constants $D_{i, c}$ (Fig.3B) and exponents $\alpha_{i, c}$ (Fig.3C), which is consistent with the experiments. However, this strong variability is also strongly present in the null - homopolymer - model (Fig.3B-C), even if at a lower extent than in the mouse and in Drosophila models. This result suggests that part of the heterogeneity observed in time-averaged MSD does not stem from the multi-scale organization of chromosomes in TADs or compartments, but rather from a finite-size effect due to the limited duration of the monitored trajectories to measure the MSD (Suppl. Fig.S2).

To mitigate this confounding factor and focus on the role of structural heterogeneities on dynamical variabilities, we next computed the ensemble-averaged MSD of all monomers over $\sim 2000$, $\sim 1$ hour-long trajectories (Fig.3D-F). In the homopolymer model (Fig 3D), we observed as expected a uniform (Rousean) behavior for all monomers with $\alpha_{i} \approx 0.5$ at short time scales (<10 s) and $\alpha_{i} \approx 0.4$ at longer time scales (>100 s), typical of crumpled polymers $(44,45,57)$. For mouse and Drosophila chromosomes, simulations predicted heterogeneous, locus-dependent MSDs (Fig 3E and 3F). The distribution of diffusion constants are broad, implying a large spectrum of loci mobilities (Fig.3G). This is particularly visible in the Drosophila case (Fig.3G) where mobility may vary by up to 3-fold between two monomers. Interestingly, in Drosophila, we also predicted the distribution to be multimodal with 2 main peaks at low and high mobility, which is fully consistent with several experiments $(23,28,36)$. The distributions of diffusion exponents per locus $\alpha_{i}$ at different time scales (Fig.3H-I) also 
support the strong heterogeneities observed in diffusion behaviors. While at short time scales $(<3 \mathrm{~s})$ exponents are rather homogeneous $\left(\alpha_{i} \approx 0.5\right)$, at larger time scales $(>10 \mathrm{~s}) \alpha_{i}$ becomes highly locus-specific and may strongly vary as a function of time. For example, at $1000 \mathrm{~s}, \alpha_{i}$ varies between 0.25 and 0.65 in Drosophila and between 0.3 and 0.5 in mice (Fig.3I). This broad range of values, including both sub- $\left(\alpha_{i}<0.5\right)$ and super- $\left(\alpha_{i}>0.5\right)$ Rousean exponents, is consistent with the large discrepancy observed experimentally in $\alpha_{i}$ across loci and conditions (Fig. 1A).

\section{Dissecting the role of compartments and TADs on chromatin motion}

The degree of dynamical heterogeneity predicted by the model can only arise from the passive interactions driving the TAD and compartment formation. Fig.4 A-C illustrate the time-evolution of the exponent $\alpha_{i}$ along the genome. As expected, we observed for the null model an overall homogeneity in dynamical parameters over time (Fig.4A). For the mouse and Drosophila models, we measured, instead, a strong heterogeneity and locus-dependence along the genome that interestingly seems to correlate with the TADs and compartments layers of organization. Many regions (Fig.4B,C) exhibit an anomalous and nonmonotonic behavior: $\alpha_{i} \sim 0.5$ at short time scales, decreases to $\sim 0.3$ (sub-Rousean regime) at intermediate time scales, increases to $\sim 0.6$ (super-Rousean regime) and then retrieves a standard crumpled polymer-like behavior $\left(\alpha_{i} \sim 0.4\right)$ at very large time scales. Interestingly, such crossovers between sub- and super-Rousean regimes or between super-Rousean and normal regimes have also been observed experimentally (see Fig.1A).

\section{Chromatin mobility is reduced in compact compartments}

To quantitatively associate such anomalous behaviors with structural features, we first separately plotted the MSD for loci in active (A) and repressive (B) compartments (Fig.4D,E). In mice (Fig.4D), both compartments have a similar - weak - degree of heterogeneity with comparable diffusion constants and exponents (Fig.4F). This confirms that the dynamical heterogeneity and difference between eu- and heterochromatic loci are usually weak in highly plastic cells as experimentally observed in mouse mESC (19) and human cancer U2OS cells (23). In Drosophila (Fig.4E), loci in the A compartment are on average more mobile than those in the B compartment with a mean increase in mobility of $\sim 60 \%$ for A monomers (Fig.4G left panel). In fact, the A monomers correspond to the high mobility peak observed in Fig.3G. This is consistent with the observation that differentiated cells (like Kc167) exhibit significant differences in mobility between active and repressive regions $(19,36)$. We also observed that the distribution of exponents is much broader for B than for A monomers (Fig.4G right panel), suggesting globally more heterogeneity and more loci with anomalous behaviors in the $B$ compartment. However, not all the loci in B have anomalous dynamics (Fig.4E right panel) and all types of behaviors present in the general population of monomers (Fig.3F) are observed in the B compartment. This suggests that compartmentalization per se is not the main driving force of heterogeneity in our model. 

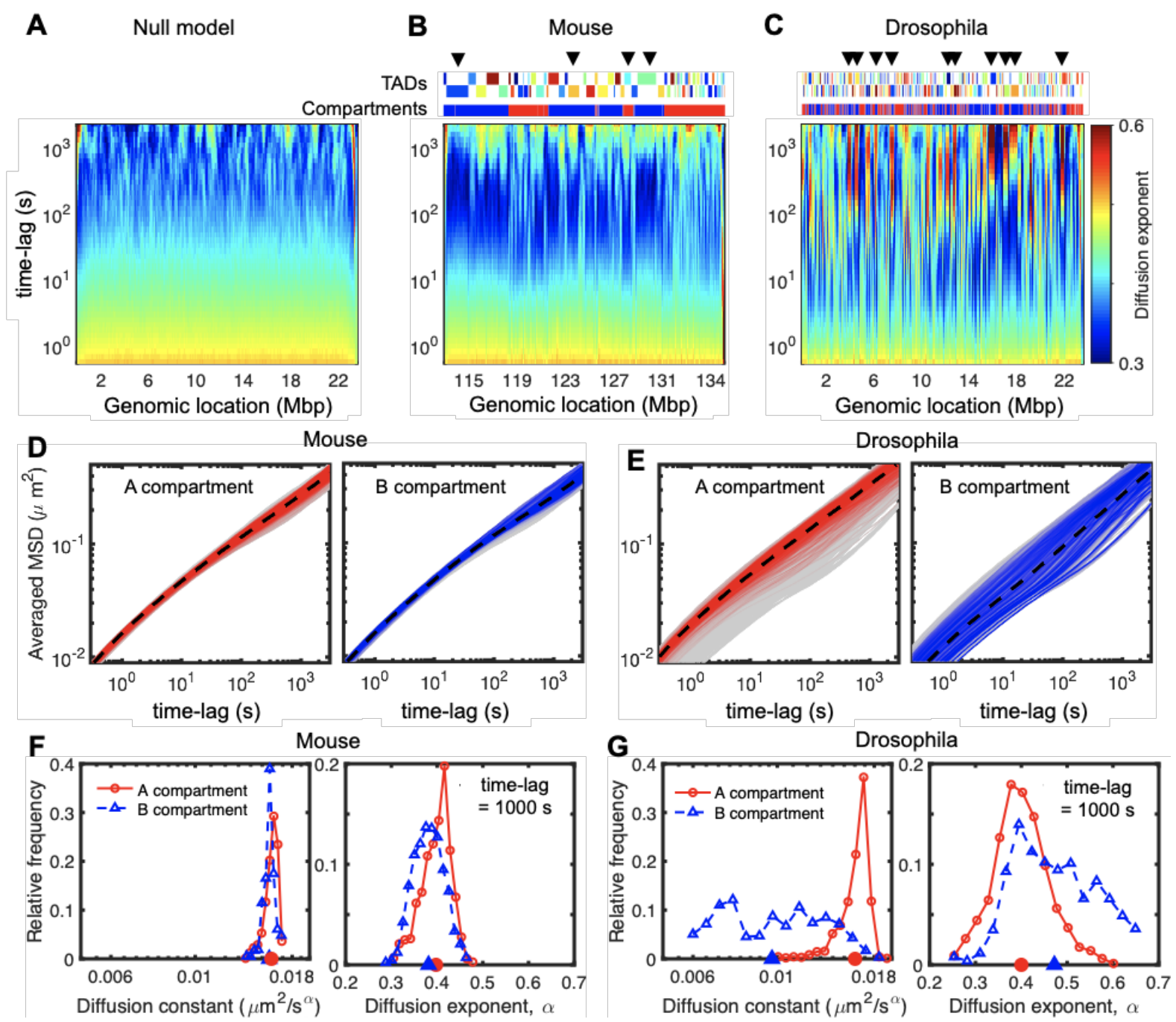

Fig 4: Heterogeneity in dynamics and its relation with compartments. (A-C) Time-evolutions of diffusion exponents along the genome for null model (A), mouse (B) and Drosophila (C), calculated by the derivatives of logarithmic MSD curves (Fig. 2G-2I and Methods). The upper tracks in (B) and (C)) show the corresponding TADs and compartments, and arrows indicate some anomalous behavior (nonmonotonic evolutions) regions. (D) MSD curves of the monomers in A (left panel) and B (right panel) compartments in the mouse case. The dashed black curves show the averaged over all loci in the same compartments, and gray shaded areas are MSDs of all monomers. (E) As in (D) for the Drosophila model. (F) The distributions of diffusion constant (left panel) and exponent (right panel) at $1000 \mathrm{~s}$ time-lag for the monomers in A (red) and B (blue) compartments in the mouse case. Their averaged values are indicated on the horizontal axis. (G) The same analysis as in (F) is shown for Drosophila simulations.

\section{Anomalous behavior is associated with TAD compaction}

We thus reasoned that TADs may be a good suspect for driving the anomalous, nonmonotonic diffusion observed in our heteropolymer models. To address this, we first selected two TADs, one from Drosophila (size $\sim 480 \mathrm{kbp}, 50 \%$ reduction in volume compared to a region of similar size in the null model) and one from mouse (size $~ 1.52 \mathrm{Mbp}, 30 \%$ volume reduction) with different degrees of compaction and different sizes (Fig.5A,C). For the mouse TAD (Fig.5A,B) which is bigger but less compact, anomalous behavior is weaker and most of the monomers follow a null model-like behavior. For the Drosophila TAD (Fig.5C) which is smaller but more compact, we observed that all loci inside the TAD move with an anomalous dynamics (Fig 
5D). By comparing the local Hi-C map and the time-evolution of the diffusion exponent (Fig 5C), we also remarked that the small neighboring TADs are less impacted. This suggests that smaller TADs may be less prone to heterogeneity. Since one main difference between mouse and Drosophila heteropolymer models is the intra-TAD strength of interaction (see Table 2 and Fig.2J), these observations point towards an important role played by the intra-TAD compaction level on driving anomalous behaviors (Suppl. Fig. S1).

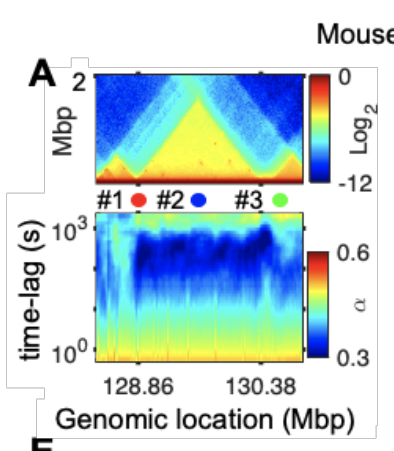

E

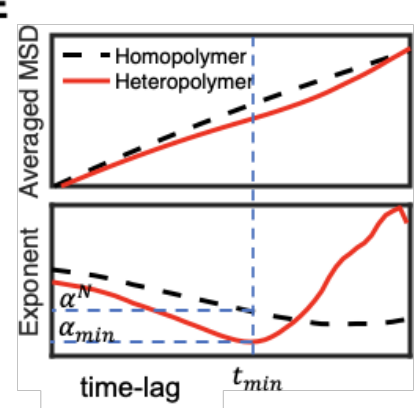

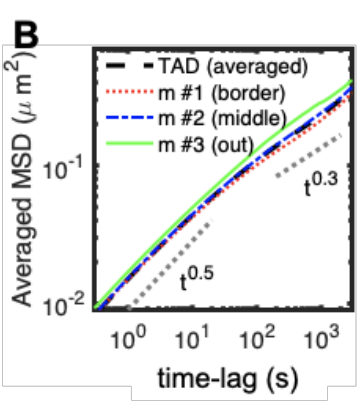

$\mathbf{F}$

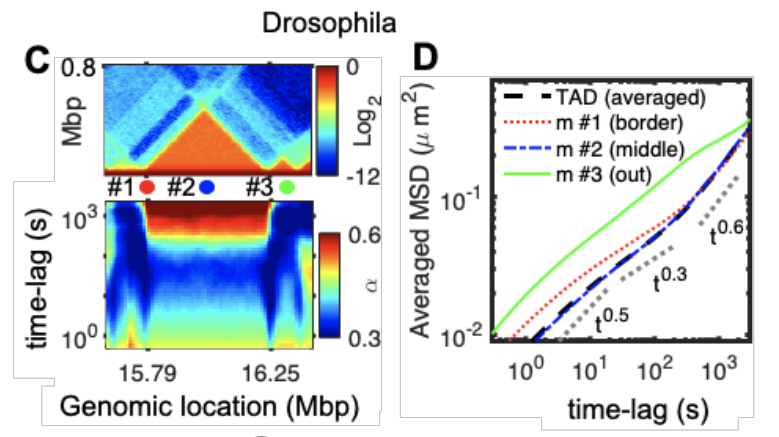

G
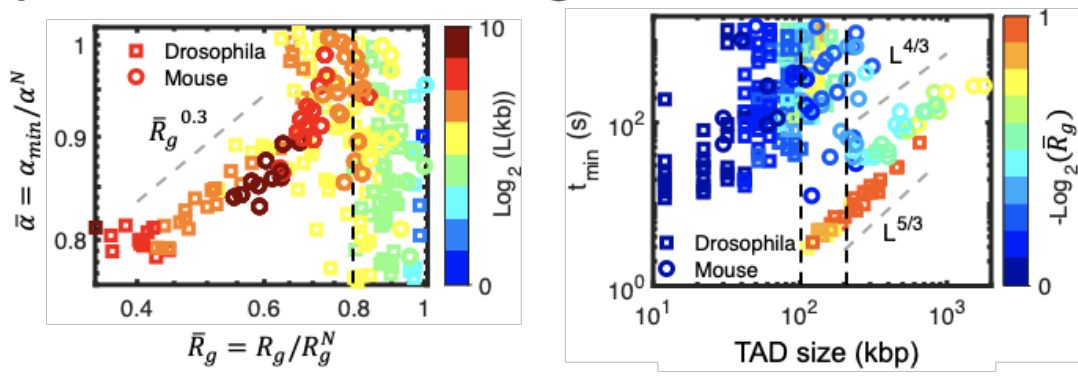

Fig 5: Compaction and highly dense regions leads to anomalous behavior. (A) Comparison of $\mathrm{Hi}-$ $\mathrm{C}$ map (top) and the corresponding time-evolution of exponent (bottom) for the mouse chr1:128.36130.88 Mbp region, which includes a 1.52 Mbp-long TAD. (B) MSD for three monomers annotated in panel (A) (red: $m \# 1$ at the border of TAD, blue: $m \# 2$ at the middle of the TAD, green: $m \# 3$ outside the TAD) and average MSD for all the monomers inside the TAD. (C-D) As in (A-B) but for the Drosophila chr2L:15.64-16.4 Mbp region, which includes a $460 \mathrm{~kb}$-long TAD. (E) Typical average MSDs for a TAD predicted by the homopolymer and heteropolymer models (top), and their corresponding derivatives in logarithmic scales (bottom). From these plots, we extracted the minimum exponent $\alpha_{\min }$, the corresponding time $t_{\text {min }}$, and expected exponent from homopolymer model $\alpha^{N}$. (F) $\bar{\alpha}_{\text {min }}\left(=\alpha_{\min } / \alpha^{N}\right)$ against $\bar{R}_{g}$ for drosophila and mice. The symbols are colored by the TAD size, $L$. (G) $t_{\text {min }}$ as a function of TAD size for drosophila and mice. Symbols are colored by the compaction level of TADs $\left.-\log _{2} \overline{(R}_{g}\right)$.

To quantify this, we considered, for each TAD, 3 'structural' quantities: $L$, the TAD size; $R_{g}$, its radius of gyration; $R_{g}^{N}$, the radius of gyration for a domain of size $L$ in the null model. Strongly compacted TADs are characterized by low values for $\bar{R}_{g} \equiv R_{g} / R_{g}^{N}$. And we introduced 3 'dynamical' observables (Fig.5E): $\alpha_{\text {min }}$, the minimum diffusion exponent value over time computed from the average MSD of monomers inside the TAD; $t_{\text {min }}$, the time when $\alpha_{\text {min }}$ is reached; $\alpha^{N}$ the exponent of the null model at $t_{\min }$. The strength of the anomalous behavior is thus captured by the ratio $\bar{\alpha}_{\text {min }} \equiv \alpha_{\min } / \alpha^{N}$ (low ratios $<1$ corresponding to strong anomality), and its duration by $t_{\min }$.

We found that $\bar{\alpha}_{\text {min }}$ is an increasing function of $\bar{R}_{g}$ for $\bar{R}_{g} \leq 0.8$ while we do not observe a clear-cut dependence of $\bar{\alpha}_{\text {min }}$ on $L$ (Fig.5F). Interestingly, Drosophila and mouse TADs follow 
the same scaling law $\left(\bar{\alpha}_{\min } \sim \bar{R}_{g}{ }^{0.3}\right)$, suggesting that compaction level is the main driver of the strength of the anomalous behavior. For weakly compacted domains $\left(\bar{R}_{g}>0.8\right)$ or small TADs, the value of $\bar{\alpha}_{\text {min }}$ is very fluctuating. We also observed that, beyond a critical size ( $\sim 100 \mathrm{kbp}$ for drosophila and $\sim 200 \mathrm{kbp}$ for mouse), $t_{\min }$ evolves as $\sim L^{5 / 3}$ for the drosophila case and $\sim L^{4 / 3}$ for the mouse case, the role of $\bar{R}_{g}$ being less clear (Fig.5G). These results show that the duration of the anomalous, nonmonotonic diffusion in heteropolymer models depends on the TAD size and compaction, longer domains being impacted for longer times. As for $\bar{\alpha}_{\text {min }}$, we found that $t_{\min }$ values for small TADs are very dispersed with no correlation with TAD size.

\section{Anomalous behavior emerges from a crossover towards collective motion}

A
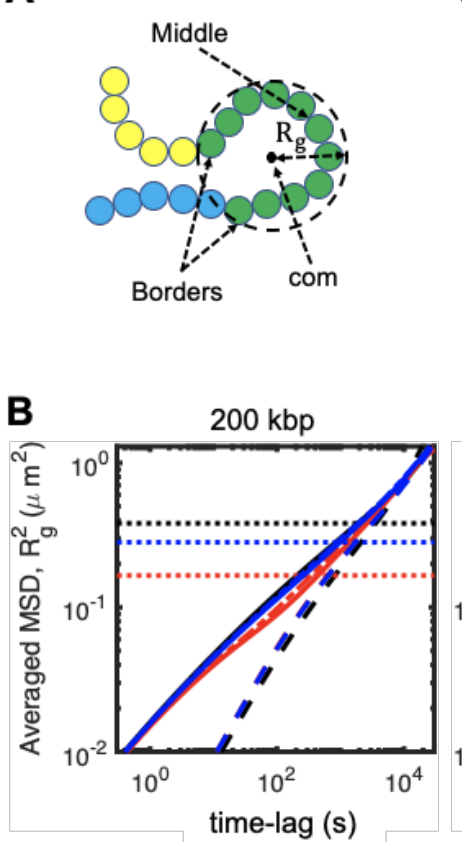

C

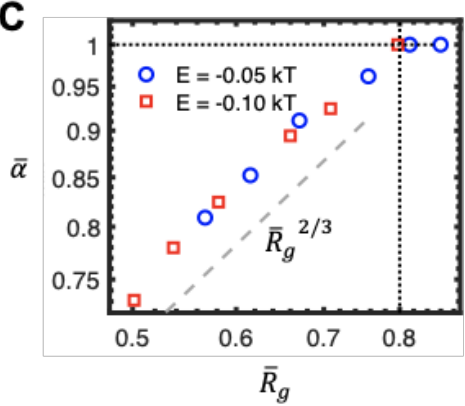

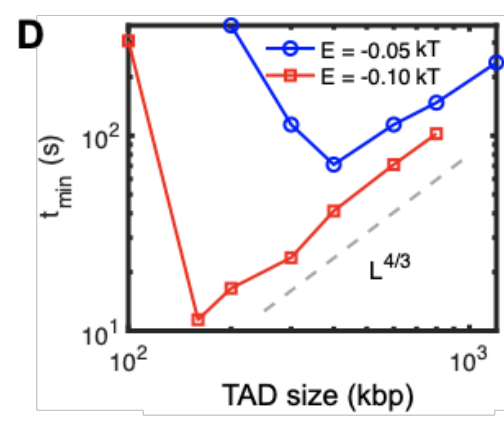

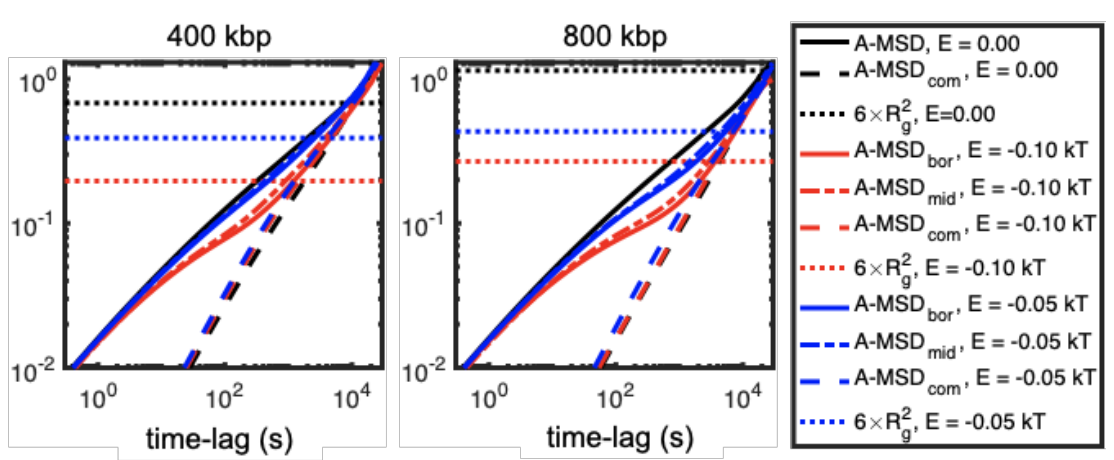

Fig 6: Anomalous behavior of uniform models. (A) A cartoon to depict 'middle' and 'border' monomers, along with the center of mass and the radius of gyration of a TAD. (B) Comparison between MSD of the monomers in the middle (mid) and borders (bor) of TAD and of the center of mass of the TAD (com) (see panel B) from uniform heteropolymer models with TAD size of $200 \mathrm{kbp}$ (left), $400 \mathrm{kbp}$ (middle) and $800 \mathrm{kbp}$ (right) for two different intra-TAD strength of interaction (black: 0kT/null model, blue: $-0.05 \mathrm{kT}$, red: $-0.1 \mathrm{kT})$. The spatial size $\left(6 R_{g}^{2}\right)$ for the different strengths of interactions is shown as dotted lines. (C,D) $\bar{\alpha}_{\min }$ as a function of $\bar{R}_{g}$ and $t_{\min }$ as a function of the TAD size $L$ for the uniform models and for different strengths of interaction.

The existence of anomalous behaviors with MSDs exhibiting transitions between different - a priori opposite (sub- vs super-Rousean) - regimes is thus strongly associated with TAD compaction. To go deeper in the analysis of this association and to better understand how these transitions emerge from compaction, we introduced toy heteropolymer models (see Methods) where we can independently play with the TAD size and intra-TAD strength of interaction. We considered simplified 'uniform' models where genomes are partitioned into adjacent TADs of the same size (see Methods and Suppl. Fig. S3). For a given TAD size and intra-TAD strength of interaction, all monomers of the chain have very similar MSDs 
(Fig.6B), with only a weak positional effect translating the bead positioning inside the TAD, consistent with our observations made on more 'complex' models (Fig.5B,D). As expected, emergence of anomalous behavior occurs for larger TADs and stronger interactions (Fig.6B and Suppl. Fig S4) where compaction starts to be substantial (Suppl. Fig. S5). Merging all the investigated TAD sizes and strength of interactions together, we confirmed that $t_{\text {min }}$ increases with $L$ (Fig.6D) and that below a given critical compaction level $\left(\bar{R}_{g} \leq 0.8\right), \bar{\alpha}_{\text {min }}$ is an increasing function of $\bar{R}_{g}$ (Fig.6C). The dependency is steeper $\left(\bar{\alpha}_{\min } \sim \bar{R}_{g}{ }^{2 / 3}\right.$ ) in the uniform models than for the more heterogeneous mouse and drosophila cases. In the uniform models, for small and weakly compacted TADs, we observe $\bar{\alpha}_{\min } \sim 1$. This suggests that the dispersion of $\bar{\alpha}_{\min }$ values observed at this compaction regime $\left(\bar{R}_{g}>0.8\right)$ in the heterogeneous drosophila or mouse models (Fig.5F) reflects in fact the influence of neighbor - more compacted - TADs on these - normally weakly-impacted - domains (Suppl. Fig. S6).

Interestingly, by plotting the MSD of the center of mass of TADs in the uniform models ( $M S D_{\text {com }}$ ), we observed that, in the cases of strong anomalous dynamics, the MSD of individual monomers $M S D_{i}$ follows the dynamics of the center of mass in the super-Rousean regime (Fig.6B). Since $M S D_{\text {com }}$ does not depend strongly on the intra-TAD strength of interactions, the transition between the small time-scale - homopolymer-like - diffusion and the large timescale - center-of-mass-like - regime is driven by the degree of compaction of the TAD. The cross-over time between this two regimes corresponds typically to the Rouse time of the TAD (58), ie the typical time for the center of mass of the TAD to diffuse over the typical 3D physical size of the TAD (given by the end-to-end distance $\sim \sqrt{6} R_{g}$ ) (Fig.6B). Overall, these results suggest that the non-monotonic - anomalous - evolution of $\alpha_{i}$ represents a crossover between the 'fast' diffusion of single monomer at short times and a 'slow', collective regime where loci of the TADs move coherently at longer time-scales.

\section{Coherent motion and slow dynamics of intra-TAD monomers}

We then reasoned that such coherent moves might lead to correlations in the motion of monomers. We thus computed from our simulations the correlations $C_{i j}(\Delta t)$ between the displacement of two monomers $i, j$ during a given time lag $\Delta t$ (see Methods). Intuitively, this quantity describes how correlated are the motions of two loci relative to the center of mass of the system. Fig 7A shows the normalized pair-correlation matrix $\left(C_{i j} / C_{i i}\right)$ at different time-lags. For the null, homopolymer model (Fig.7A), correlations decay uniformly as the genomic separation increases between the two loci (Fig.7B). The typical genomic distance $s_{\text {corr }}$ up to which two monomers are correlated augments with the time-lag from a few dozens of kbp for second-scale displacement to Mbp after hours. This is a direct consequence of the polymeric nature of chromosomes and of the conserved connectivity along the chain. For the mouse and the Drosophila cases, we observed patterns in the correlation matrices that are strongly related to the TAD organization as already seen by (41), more compact TADs (drosophila case) being more impacted (Fig.7A). This observation confirms the coherent motion of monomers inside TADs and its relation to intra-TAD compaction (Figs.5,6). Interestingly, for longer time-lags, correlations between monomers of the same compartment become significant, in particular in the mouse case where compartments are more segregated (Fig.2FI). Overall, this leads to larger $s_{\text {corr }}$ values (Fig.7B and Suppl. Fig. S7), the difference with the null model increasing with the time-lag. 
A
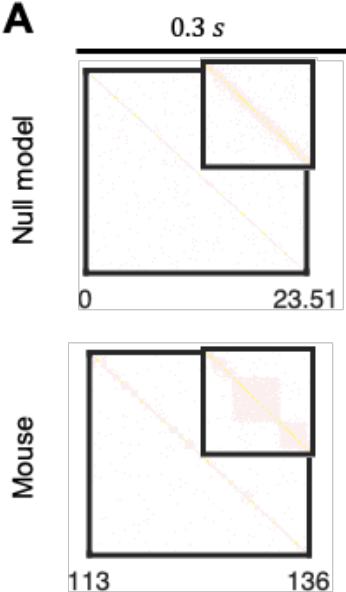

$\prod_{113}$

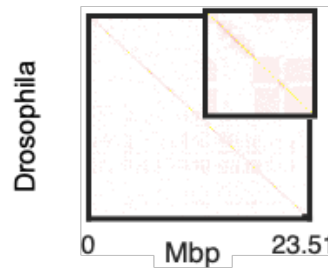

$30 s$

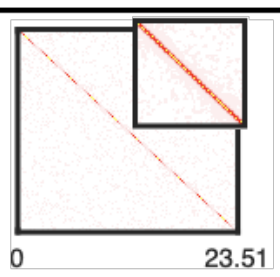

23.51
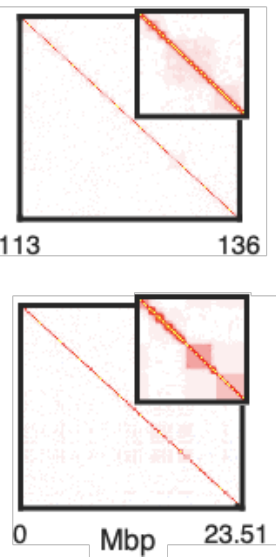

$3000 s$ time-lag
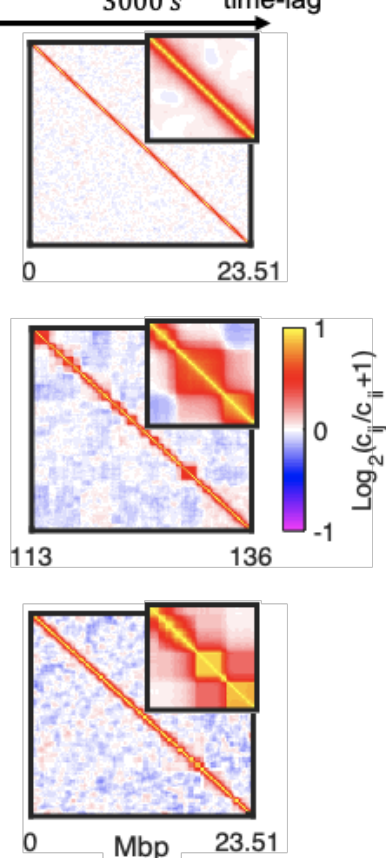

B
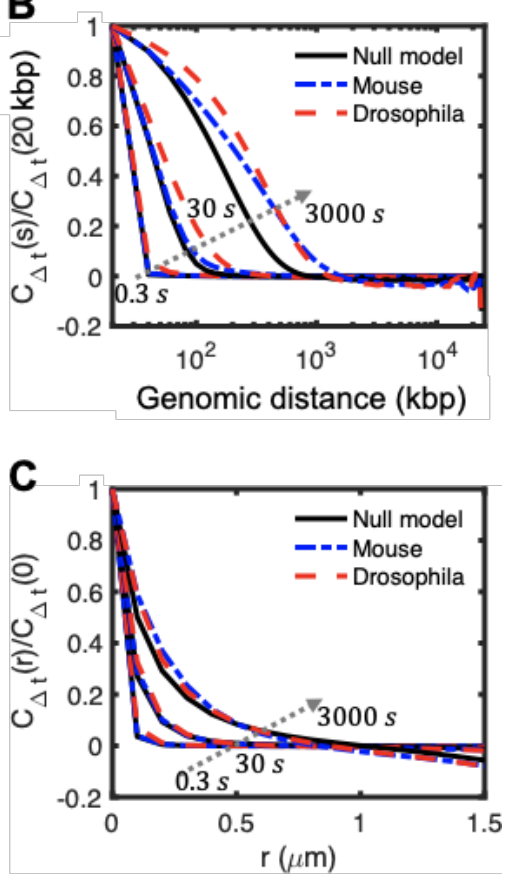

Fig 7: Spatiotemporal correlations of loci displacements. (A) Normalized matrices of pair correlation of motions with respect to the motion of center of mass (i.e. fixed cells) for null model (top panels), mouse (middle panel) and Drosophila (bottom panels) at different time-lags. Insets represent zooms of the $2 \mathrm{Mbpx} 2 \mathrm{Mbp}$ central parts of the matrices. (B-C) Normalized correlations as a function of genomic distance (B) and spatial distance (C) for different time-lags same as panel (A). Arrows indicate increasing time-lag.

Additionally, we looked at the spatiotemporal correlation function $C_{\Delta t}(r)$ (Methods) that represents the average correlation between the displacement of two monomers separated by a 3D distance $r$ during a given time lag $\Delta t$ (44) (Fig.7C), a quantity more easily accessible experimentally (24). We found that the typical size of the spatial regions with correlated motions increases with the time-lag from $\sim 100 \mathrm{~nm}$ for second-scale displacement to $\sim 1 \mu \mathrm{m}$ after hours. These predictions remain largely underestimated compared to experiments (few microns already after a few seconds) (24). Surprisingly, we also did not find strong differences between the null model and the mouse and drosophila cases, demonstrating that $C_{\Delta t}(r)$, which is an average over all the loci, does not capture the effect of compaction on correlated motion. All this suggests that the larger spatiotemporal correlations observed experimentally are not the signature of TAD formation or A/B compartmentalization.

To further dissect the correlated motion of intra-TAD monomers, we tracked the stochastic dynamics of the spatial distance between all pairs of monomers separated by $100 \mathrm{kbp}$ along the chain in the null and drosophila cases (Suppl. Fig. S8). From each pair and each trajectory, we extracted three quantities (Fig.8A) (45): the first passage time $\tau_{\text {first }}$ which corresponds to the first time the two loci encounter each other (i.e. when the distance goes below $d_{c}=200 \mathrm{~nm}$ ); the residence time $\tau_{r}$ which defines duration the two loci remain closer than $d_{c}$; and the search time $\tau_{s}$ which is the time interval between two consecutive encounters. In the drosophila case, we sorted pairs as intra-TADs if both monomers belong to the same TAD or as inter-TADs otherwise. For $\tau_{\text {first }}$, intra-TAD pairs tend to meet more rapidly (average $\sim 10 \mathrm{~s}$ ) than inter-TAD and null model pairs (average $\sim 50 \mathrm{~s}$ ) (Fig.8B). The distribution of intraTAD $\tau_{\text {first }}$ exhibits a scaling law while inter-TAD and null model pairs distribution is 
exponential. Residence times $\left(\tau_{r}\right)$ are exponentially distributed for all pairs with a decay time 3-fold larger for intra-TAD pairs ( $\sim 6$ for intra-TAD vs $\sim 2 \mathrm{~s}$ for inter-TAD and null model pairs) suggesting stable contacts and a slow relative dynamics between intra-TAD monomers (Fig.8C) . The distributions of $\tau_{s}$ are almost identical for inter-TAD and null model pairs with an exponential decay characterized by long waiting times ( 40 s) (Fig 8D). Intra-TAD search times are much smaller (average value $\sim 6 \mathrm{~s}$ ) and characterized by a power-law.
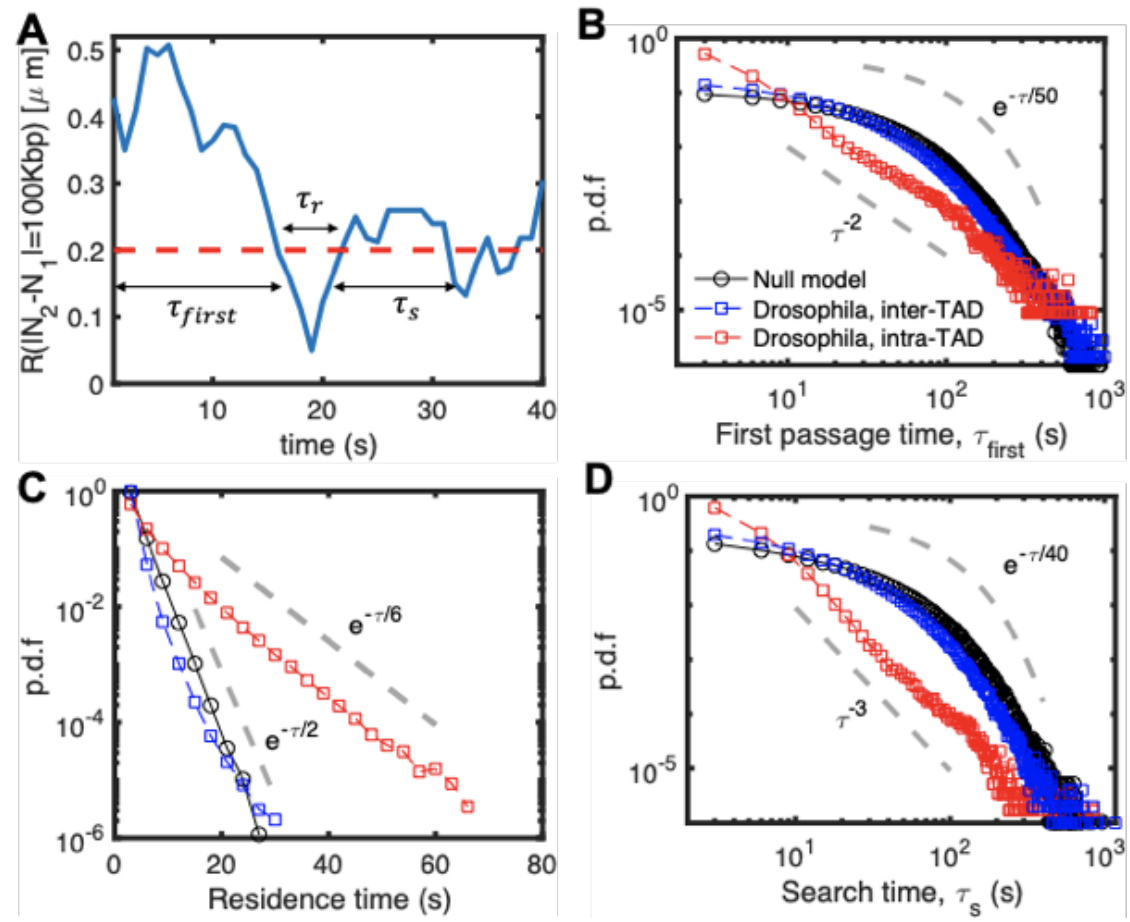

Fig 8: Intra-TAD slow dynamics. (A) Typical time-evolution of the distance between two monomers separated by $100 \mathrm{kbp}$. The arrows illustrate the first passage time $\tau_{\text {first }}$, the residence time $\tau_{r}$, and search time $\tau_{s}$. The red dashed line represents the cut-off distance $d_{c}=200 \mathrm{~nm}$. (B-D) Probability distribution functions (p.d.f) for $\tau_{\text {first }}(\mathrm{B}), \tau_{r}(\mathrm{C})$ and $\tau_{s}(\mathrm{D})$ in the homopolymer case (black) and for Drosophila intra-TAD (red) and inter-TAD (blue) pairs of monomers. The grey dashed lines represent power-law or exponential fit. Note that in panels B and D, p.d.fs are shown in a log-log plot while in panel $C$ it is in a log-lin plot.

\section{Discussion and Conclusion}

Experiments probing chromatin motion have highlighted the large heterogeneity existing inside cell nuclei and across biological conditions and have suggested that chromatin may behave sometimes like a liquid sometimes like a gel. In this paper, we investigated chromosome dynamics using biophysical modeling in order to interpret, in a unified framework, how these different physical states and dynamical heterogeneities may emerge from the same first principles driving genome folding.

Based on a mechanistic polymer model that captures the main structural properties of chromosome organization, we were able to quantify the motion of chromatin at the chromosome territory level. Previous similar approaches on human chromosomes $(40,41,44)$ have shown that such types of heteropolymer models are consistent with a heterogeneous, sub-Rousean, (A/B) compartment-dependent and spatially-correlated chromatin dynamics. To go beyond these works $(40,41,44)$ and get a broad view of chromatin motion on contrasted situations, we studied in parallel 3 cases: a reference homopolymer model, and two 
heteropolymer models of a less compact, stem-cell-like chromatin (mESC) and a more compact, differentiated-cell-like organization (drosophila Kc167).

Our results demonstrate indeed the heterogeneous locus-dependency of chromatin motion. We found that this heterogeneity reflects the various degree of compaction that may exist along the genome: while loci inside small or weakly-compacted TADs (or compartments) exhibit a rather homogeneous, fast, Rouse-like diffusion, loci inside compact TADs have a lower mobility and experience crossovers between different diffusion regimes (from Rousean to sub-Rousean to super-Rousean to Rousean modes). Using uniform models, we demonstrated that such anomalous dynamics is the signature of collective, coherent motion at the level of the strongly-compacted region.

We observed that the existence of 3D chromatin domains of correlated displacements, the socalled dynamically-associated domains (DADs) $(24,26)$, emerges intrinsically from the polymeric nature of chromatin. However, the persistence of these domains is strongly related to the 3D chromosome organization: loci in the same TAD and, to a lesser extent, in the same compartment are more likely to be in the same DAD, as also observed by Di Pierro et al for human chromosomes (41).

In our heteropolymer models, we integrated the multiple layers of chromatin organization using passive interactions, i.e. ATP-independent processes that satisfy detailed balance (59). It is interesting to note that using such interactions, we can capture super-Rousean regimes, not as a consequence of directed forces or active processes but as a crossover regime between a slow, coherent mode of motion at slow or intermediate time-scales to a normal Rouse-like dynamics at longer time-scales. However, our analysis reveals that passive interactions only cannot quantitatively capture the fast and large-scale average increase of correlated motion across the nucleus $(24,60)$, suggesting that active processes mediate this large-scale growth in spatial correlations (44), putatively via the actions of extensile motors on chromatin mediated by hydrodynamics interactions (61) or crosslinks and interactions with the nuclear membrane (62). Active chromatin-loop extrusion by cohesins or condensins $(63,64)$, a main driver of TAD formation in mammals $(65,66)$, may also act on chromatin motion (67) either under the same phenomenology as we described here, i.e. by generating loops and compacting chromatin; or by boosting locally the mobility on a short-time scale corresponding to the loop extruder residence time at a locus (68).

To conclude, our work provides a unified framework to rationalize the wide variety of behaviors observed experimentally. Heterogeneity in the diffusion and exponent constants are driven by heterogeneity in TAD organization and chromatin condensation. The observed fluid-like behavior $(49,50)$ of chromatin is likely to be associated with weakly-compacted, dynamic chromatin. This would typically correspond to stem-cell-like conditions where $A$ and $B$ compartments are still not entirely formed and where architectural proteins driving their organization like HP1 (69) are not fully loaded (70). This may explain why no clear differences are globally observed between eu- and heterochromatin in U2OS (23), a highly plastic and transformed human cell line. The gel-like state of chromatin (22) is associated with stronglycompacted regions which, in our examples, mainly correspond to TADs but would also be observed for any chromatin structure with similar degrees of compaction. Its dynamic signature relies on its low mobility, the presence of local coherent motion with slow internal dynamics and crossover between different diffusion regimes for individual loci (see above). Interestingly, Khanna et al (22) have described a gel state at the immunoglobulin heavy chain locus in pro-B cells, a highly compacted region. They observed very slow, sub-Rousean, 
internal motion between $\mathrm{V}_{\mathrm{H}}$ and $\mathrm{D}_{\mathrm{H}} \mathrm{J}_{\mathrm{H}}$ segments and a crossover between super-Rousean and Rousean regime for individual MSD of the $D_{H} J_{H}$ segment, which is consistent with our analysis of strongly self-interacting regions (Suppl. Fig. 9). At the nuclear scale, this gel state could be only localized on few compacted regions like on centromeres or telomeres which exhibit MSDs with crossovers even in U2OS cells (27). However, we expect this state to become more and more predominant as cell differentiation progresses and compartments like heterochromatin achieved their final compaction state (36). This may lead in extreme cases to a solid state with an almost arrested dynamics of chromatin as recently suggested by FRAP analysis on euand heterochromatin regions of mouse embryonic fibroblast cells (51).

A better characterization of the regulation of the fluid, gel or solid state of chromatin motion would require the development of upgraded polymer models integrating the main passive and active processes driving genome folding but also the development of new experimental approaches allowing to quantify simultaneously the dynamics of many loci (whose genomic positions are known) at high spatial and temporal resolution. Recent progresses in multiple loci $(71)$ and in super-resolution $(16,25,72,73)$ live imaging would make it possible to test quantitatively the relations between mobility, coherent motion and compaction predicted by our polymer models and the role of basic mechanisms such as phase separation or loop extrusion in regulating chromosome dynamics. 


\section{Methods}

\section{Analysis of $\mathrm{Hi}-\mathrm{C}$ data}

The experimental $\mathrm{Hi}-\mathrm{C}$ datasets were downloaded from the sequence read archive (SRA) (Table 1) using fastq-dump (https://github.com/ncbi/sra-tools/wiki). Each experiment has been processed (i.e. FASTQ quality checks, Mapping to reference genomes, Filtering to remove non-informatic reads, and Merging data together) through the TADbit pipeline (https://github.com/3DGenomes/TADbit) (74). Then, we normalized data using the Vanilla method at $10 \mathrm{kbp}$ resolution (2). After generating the Hi-C map, we employed the IC-Finder tool to find TAD boundaries (75). The A/B compartments have been identified by the hic_data.find_compartments tool from the TADbit suite, using Principal Component Analysis (PCA) of the observed/expected matrices. Finally, we find chromatin loops by using hicDetectloops tool from the HiCExplorer package (https://github.com/deeptools/HiCExplorer) (76).

Table 1: Hi-C datasets used in this study. Data for Mouse ES cell and Drosophila melanogaster Kc167 were published in (77) and (78), respectively. Dataset, GEO accession number, organism, cell type, restriction enzyme, number of independent runs, and reference genome to map data are given.

\begin{tabular}{|l|c|c|c|c|c|c|}
\hline Dataset & $\begin{array}{c}\text { GEO } \\
\text { accession }\end{array}$ & Organism & Cell type & $\begin{array}{c}\text { Restriction } \\
\text { enzyme }\end{array}$ & Runs & $\begin{array}{c}\text { Reference } \\
\text { genome }\end{array}$ \\
\hline$(77)$ & GSM2533818 & $\begin{array}{c}\text { Mus } \\
\text { musculus }\end{array}$ & $\begin{array}{c}\text { mESC } \\
(\mathrm{E} 14)\end{array}$ & Dpnll & 14 & $\mathrm{~mm} 10$ \\
\hline$(78)$ & GSM3346506 & $\begin{array}{c}\text { Drosophila } \\
\text { melanogaster }\end{array}$ & $\begin{array}{c}\text { Embryo } \\
(\text { Kc167) }\end{array}$ & Dpnll & 2 & $\mathrm{dm6}$ \\
\hline
\end{tabular}

\section{Polymer models and simulations}

Each genomic segment under investigation is modeled as a semi-flexible self-avoiding polymer in which one monomer consists of $2 \mathrm{kbp}$ of genome and has a diameter of $50 \mathrm{~nm}$ (Fig 2A). The chain is moving on a FCC lattice under periodic boundary conditions to account for confinement by other genomic regions, as described in (45).

The Hamiltonian of a given configuration is given by

$$
H=\kappa \sum_{i=2}^{N-1} \quad\left(1-\cos \theta_{i}\right)+\sum_{i, j} \quad\left(E_{c(i), c(j)}+E_{T A D, c(i)} \delta_{t(i), t(j)} \delta_{c(i), c(j)}+E_{L o o p} \delta_{l(i), l(j)}\right) f_{i j},
$$

with $f_{i j}=1$ if monomers $i$ and $j$ occupy nearest neighbor sites on the lattice ( $=0$ otherwise). The first term in $H$ accounts for the bending rigidity of the chain with $\kappa$ the bending stiffness and $\theta_{i}$ the bending angle between monomers $i-1$, iand $i+1$. The second term refers to contact interactions driven by the compartment $(c(i))$, TAD $(t(i))$ or loop $(l(i))$ state of each monomer. $E_{c(i), c(j)}$ (either $E_{A A}, E_{B B}$ or $E_{A B}$ ) accounts for compartment-compartment interactions, $E_{T A D, C(i)}$ (either $E_{T A D, A}$ or $E_{T A D, B}$ ) for intra-TAD interactions, $E_{\text {Loop }}$ for CTCF-mediated loop interaction and $\delta_{m, n}=1$ if $m=n$ (=0 otherwise). In the homopolymer model, all interaction parameters except $\kappa$ were set to zero.

The lattice volumic fraction $(\sim 0.5)$ and bending energy $(1.2 \mathrm{kT})$ were chosen to simulate the coarse-grained dynamics of a chromatin fiber of Kuhn length $\sim 100 \mathrm{~nm}(31,79)$ and of bpdensity $\sim 0.01 \mathrm{bp} / \mathrm{nm}^{3}$, typical of mammalian and fly nuclei (80). Dynamics of the chain was 
simulated using kinetic Monte-Carlo, starting from unknotted initial configurations, as detailed in (45). Each Monte-Carlo step (MCS) consists of N (total number of monomers) local moves including reptation moves. Such implementation has been shown to well reproduce the structural and dynamical properties of long, confined polymers $(45,81)$. For each simulation, we discarded the first $10^{6} \mathrm{MCS}$ to reach steady state and then stored snapshots every $10^{2}$ MCS during $10^{7}$ MCS. For each situation (homopolymer, mouse and fly heteropolymer models), 20 different independent trajectories were simulated starting from different initial configurations.

\section{Parameter inference of the heteropolymer models}

To infer parameters in both (mouse and drosophila) cases, we first mapped TADs, compartments and loops extracted from the experimental $\mathrm{Hi}-\mathrm{C}$ map into the heteropolymer model and then iteratively adjusted the interaction parameters to optimize the correspondence between the predicted and experimental $\mathrm{Hi}-\mathrm{C}$ maps. For simulations, we estimated the contact frequency between any pair $(i, j)$ of monomers as the probability to observe $i$ and $j$ at a distance less than a cutoff value $r_{c}$ in all our snapshots. To quantitatively compare simulated contact probabilities to experimental $\mathrm{Hi}-\mathrm{C}$ map, we transformed the experimental contact frequencies $\left(H_{i j}\right)$ to contact probabilities $\left(P_{i j}\right)$ using the following relation $P_{i j}(\exp )=\min \left(1, \frac{H_{i j}}{\underline{H}_{ \pm 1}}\right)$, where $\underline{H}_{ \pm 1}$ is the median value of $\left\{H_{i, i+1}\right\}$ (7). Our criteria for finding the optimal parameter values (energies $E_{A A}, E_{B B}, E_{A B}, E_{T A D, A}, E_{T A D, B}, E_{\text {loop }}$ and cutoff distance $r_{c}$ ) were the maximization of the Pearson correlation between matrices and the minimization of the $\chi^{2}$ score, $\chi^{2}=$ $\frac{1}{N} \sum_{i, j} \frac{\left[P_{i j}(\operatorname{sim})-P_{i j}(\exp )\right]^{2}}{\sigma_{i j}^{2}(\exp )}$, where $N$ is total number of $\{i, j\}$ and $\sigma_{i j}^{2}(\exp )$ is the experimental standard deviation on $P_{i j}(\exp )$ estimated over experimental replicates. The optimization was done using a grid-search algorithm over the parameter values in the range [-0.3:0] kT for energy parameters and in the range of [70:200] $\mathrm{nm}$ for $r_{c}$. In Table 2 we summarized the optimal values for each case with their corresponding $\chi^{2}$ and Pearson correlation.

Table 2: Optimized parameter sets and corresponding Pearson correlation and $\chi^{2}$ values for Mouse chromosome 1:113-136 Mbp and Drosophila chromosome 2L.

\begin{tabular}{|c|c|c|c|c|c|c|c|c|c|}
\hline \multirow{2}{*}{ Model } & \multicolumn{5}{|c|}{ Energies (kB $\mathbf{T})$} & \multirow{2}{*}{$r_{c}(n m)$} & $\chi^{2}$ & $\begin{array}{c}\text { Pearson } \\
\text { corr. }\end{array}$ \\
\hline & $E_{A A}$ & $E_{B B}$ & $E_{A B}$ & $E_{T A D, A}$ & $E_{T A D, B}$ & $E_{L o o p}$ & & & 0.95 \\
\hline Mouse & -0.02 & -0.03 & 0.00 & -0.06 & -0.04 & -0.30 & 141 & 0.06 & 0.95 \\
\hline Drosophila & -0.05 & -0.10 & -0.05 & -0.10 & -0.10 & 0.00 & 106 & 0.15 & 0.87 \\
\hline
\end{tabular}

\section{Time mapping of simulations}

In kinetic Monte-Carlo ( $\mathrm{KMC}$ ) frameworks, the dynamics of particles depend on the acceptance ratio of local trial moves. For very small step size in trial moves and very weak potentials, KMC results are equivalent with Brownian dynamics, while for longer step sizes and stronger interactions, the KMC time steps need to be rescaled (82) (83). Since we are simulating a unique chain in the box in absence of external forces, we expect the MSD $\left(g_{3}\right)$ of the center of mass of the whole polymer to be independent of the investigated model (homopolymer or heteropolymer). However, we found (Suppl. Fig. S10A) $g_{3} \cong 5.70 \times$ 
$10^{-8}\left(\mu m^{2} / M C S\right) \Delta t$ for the homopolymer model, $g_{3} \cong 5.50 \times 10^{-8}\left(\mu m^{2} / M C S\right) \Delta t$ for the mouse case and $g_{3} \cong 2.85 \times 10^{-8}\left(\mu m^{2} / M C S\right) \Delta t$ for the drosophila case, where $g_{3}$ is measured in $\mu m^{2}$ and $\Delta t$ in $M C S$. Therefore, we rescaled time $M C S \rightarrow M C S^{*}$ for each model to have similar $g_{3}$ for all cases (Suppl. Fig. S10B). The rescaled time for Mouse is $M C S^{*}=0.96$ MCS and for Drosophila $\mathrm{MCS}^{*}=0.50 \mathrm{MCS}$. As expected, the rescaled time is dependent on the strength of interaction, in a way that $M C S^{*}$ decreases for stronger interactions. Then, to translate $M C S^{*}$ to real time (sec), we compare the average simulated 2D-MSD of single loci (in $\mu m^{2}$ ), to the typical value of $0.01\left(\mu \mathrm{m}^{2} / \mathrm{sec}\right) \times \Delta t^{0.5}$ (with $\Delta t$ in sec) that has been measured in yeast (30) and that corresponds to an average MSD also observed in other species (Fig.1A). From this time mapping, we found that each $M C S^{*}$ corresponds to $\sim 3 \mathrm{msec}$ of real time.

\section{Uniform heteropolymer models}

In order to separately investigate the effects of TAD compaction, TAD arrangements and compartments, we introduce toy polymer models. In the first step, in order to explore the effects of TADs (i.e. TAD size and intra-TAD interaction), we consider "uniform" models where $20 \mathrm{Mbp}$-long chromosomal segment partitioned into consecutive TADs of uniform sizes (Suppl. Fig. S3, Suppl. Fig. S5). Then, to investigate the role of TADs arrangement, we partitioned the polymer into TADs alternating between two different sizes (Suppl. Fig. S11 upper panels, Suppl. Fig. S6). Finally, to investigate the effect of compartments, we alternatively assigned $\mathrm{A}$ and $\mathrm{B}$ compartments to the uniform TAD models with $E_{B B}<0$ and $E_{A A}=E_{A B}=0$ (Suppl. Fig. S11 bottom panels, Sup Fig. S12).

\section{Fitting of the diffusion exponent and constant}

To extract the the diffusion constant, $D_{i, c}$, and exponent, $\alpha_{i, c}$ for each trajectory $c$ and each monomer $i$, we fitted the time-average mean squared displacement $M S D_{i, c}$ by a power-law as $D_{i, c} \Delta t^{\alpha_{i, c}}$ using the Matlab function polyfit(LogMSD $\left.D_{i, c}, \log \Delta t, 1\right)$.

For the ensemble-averaged mean squared displacement $M S D_{i}$, we assumed that the diffusion exponent is a function of the time-lag $\Delta t: M_{i} D_{i}(\Delta t)=D_{i} \Delta t^{\alpha_{i}(\Delta t)}$ or, in logarithmic scale $\log M S D_{i}=\log D_{i}+\alpha_{i} \log \Delta t . \alpha_{i}(\Delta t)$ is then given by

and $D_{i}$ by

$$
\alpha_{i}(\Delta t)=\frac{\Delta \log M S D_{i}(\Delta t)}{\Delta \log \Delta t}=\lim _{\delta t \rightarrow 0} \frac{\log M S D_{i}(\Delta t+\delta t)-\log M S D_{i}(\Delta t)}{\log (\Delta t+\delta t)-\log \Delta t},
$$

$$
D_{i}=\lim _{\Delta t \rightarrow 0} M S D_{i}(\Delta t) / \Delta t^{\alpha_{i}(\Delta t)}
$$

\section{Two-dimensional density plot}

To construct the 2D density plot of chromosomes shown in Fig.2F, H, the intensity at a position $(x, y)$ is given by the sum over all monomers of a $2 \mathrm{D}$ Gaussian function mimicking the pointspread-function (PSF) of a microscope:

$$
I=\sum_{i} e^{-\frac{\left(x-x_{i}\right)^{2}+\left(y-y_{i}\right)^{2}}{2 \sigma^{2}}}
$$

with $\left(x_{i}, y_{i}\right)$ the position of monomer $i$ and $\sigma=300 \mathrm{~nm}$ chosen to get a spatial resolution of 100 $\mathrm{nm}$ in the $(x, y)$-plane. 


\section{Spatiotemporal correlation function}

We defined $C_{i j}(\Delta t)$, the pair-correlation of the displacements after a time-lag $\Delta t$ tof the monomers $i$-th and $j$-th, as $(41)$

$$
C_{i j}(\Delta t)=\left\langle\overrightarrow{\Delta r}_{i}(t ; \Delta t) \cdot \overrightarrow{\Delta r}_{j}(t ; \Delta t)\right\rangle_{t}
$$

with,

$$
\overrightarrow{\Delta r}_{m}(t ; \Delta t)=\vec{r}_{m}(t+\Delta t)-\vec{r}_{m}(t), \quad m \in i, j
$$

where $\vec{r}_{m}$ is the position of monomer $m$ with respect to the center of mass of the chain. Then, we calculated the averaged correlation $C_{\Delta t}(s)$ as a function of the genomic distance $s$ between monomers by averaging over all $C_{i j}$ with same genomic distance:

$$
C_{\Delta t}(s)=\frac{\sum_{i>j} C_{i j}(\Delta t) \delta\left(s_{i j}-s\right)}{\sum_{i>j} \delta\left(s_{i j}-s\right)}
$$

where, $s_{i j}$ is the genomic distance between monomers $i$-th and $j$-th, and $\delta(x)$ is the Kronecker delta function. Additionally, we defined the spatiotemporal correlation function (i.e., averaged correlation as function of spatial distance), $C_{\Delta t}(r)$, as (44)

$$
C_{\Delta t}(r)=\left\langle\frac{\sum_{i>j}\left[\overrightarrow{\Delta r}_{i}(t ; \Delta t) \cdot \overrightarrow{\Delta r}_{j}(t ; \Delta t)\right] \delta\left(r_{i j}(t)-r\right)}{\sum_{i>j} \delta\left(r_{i j}(t)-r\right)}\right\rangle_{t}
$$

where, $r_{i j}(t)$ is the spatial distance between monomers $i$-th and $j$-th.

Note that if we did not correct for the movement of the center of mass (Suppl. Fig. S13) we observed very long-range correlation of motions as the time-lag increases, which reflect the global motion of the center of mass.

\section{Acknowledgments}

We are grateful to Cédric Vaillant, Geneviève Fourel, Haitham Shaban, Kerstin Bystricky and the members of the Jost lab for fruitful discussions. DJ acknowledges Agence Nationale de la Recherche [ANR-18-CE12-0006-03, ANR-18-CE45-0022-01] for funding. We thank the PSMN (Pôle Scientifique de Modélisation Numérique) of the ENS de Lyon for computing resources. MDS acknowledges a STSM Grant from COST Action CA17139 supported by COST (European Cooperation in Science and Technology).

\section{Author contributions}

HS and DJ designed the research, HS and DJ performed the research, HS, MDS and DJ analyzed the data, HS and DJ wrote the paper with significant inputs from MDS.

\section{Competing interests}

The authors declare no competing interests. 


\section{References}

1. van Steensel B, Furlong EEM. The role of transcription in shaping the spatial organization of the genome. Nat Rev Mol Cell Biol. 2019 Jun;20(6):327-37.

2. Lieberman-Aiden E, van Berkum NL, Williams L, Imakaev M, Ragoczy T, Telling A, et al. Comprehensive mapping of long-range interactions reveals folding principles of the human genome. Science. 2009 Oct 9;326(5950):289-93.

3. Rowley MJ, Corces VG. Organizational principles of 3D genome architecture. Nat Rev Genet. 2018 Dec;19(12):789-800.

4. Dixon JR, Selvaraj S, Yue F, Kim A, Li Y, Shen Y, et al. Topological domains in mammalian genomes identified by analysis of chromatin interactions. Nature. $2012 \mathrm{Apr}$ 11;485(7398):376-80.

5. Wang S, Su J-H, Beliveau BJ, Bintu B, Moffitt JR, Wu C-T, et al. Spatial organization of chromatin domains and compartments in single chromosomes. Science. 2016 Aug 5;353(6299):598-602.

6. Boettiger AN, Bintu B, Moffitt JR, Wang S, Beliveau BJ, Fudenberg G, et al. Superresolution imaging reveals distinct chromatin folding for different epigenetic states. Nature. 2016 Jan 21;529(7586):418-22.

7. Szabo Q, Jost D, Chang J-M, Cattoni DI, Papadopoulos GL, Bonev B, et al. TADs are 3D structural units of higher-order chromosome organization in Drosophila. Sci Adv. 2018 Feb;4(2):eaar8082.

8. Bolzer A, Kreth G, Solovei I, Koehler D, Saracoglu K, Fauth C, et al. Three-dimensional maps of all chromosomes in human male fibroblast nuclei and prometaphase rosettes. PLoS Biol. 2005 May;3(5):e157.

9. Ou HD, Phan S, Deerinck TJ, Thor A, Ellisman MH, O'Shea CC. ChromEMT: Visualizing 3D chromatin structure and compaction in interphase and mitotic cells. Science [Internet]. 2017 Jul 28;357(6349). Available from: http://dx.doi.org/10.1126/science.aag0025

10. Ricci MA, Manzo C, García-Parajo MF, Lakadamyali M, Cosma MP. Chromatin fibers are formed by heterogeneous groups of nucleosomes in vivo. Cell. $2015 \mathrm{Mar}$ 12;160(6):1145-58.

11. Szabo Q, Donjon A, Jerković I, Papadopoulos GL, Cheutin T, Bonev B, et al. Regulation of single-cell genome organization into TADs and chromatin nanodomains. Nat Genet. 2020 Nov;52(11):1151-7.

12. Tortora MM, Salari $H$, Jost D. Chromosome dynamics during interphase: a biophysical perspective. Curr Opin Genet Dev. 2020 Apr;61:37-43.

13. Bystricky K. Chromosome dynamics and folding in eukaryotes: Insights from live cell microscopy. FEBS Lett. 2015 Oct 7;589(20 Pt A):3014-22.

14. Shaban HA, Barth R, Bystricky K. Navigating the crowd: visualizing coordination between genome dynamics, structure, and transcription. Genome Biol. 2020 Nov 17;21(1):278.

15. Bartman CR, Hsu SC, Hsiung CC-S, Raj A, Blobel GA. Enhancer Regulation of 
Transcriptional Bursting Parameters Revealed by Forced Chromatin Looping. Mol Cell. 2016 Apr 21;62(2):237-47.

16. Chen H, Levo M, Barinov L, Fujioka M, Jaynes JB, Gregor T. Dynamic interplay between enhancer-promoter topology and gene activity. Nat Genet. 2018 Sep;50(9):1296-303.

17. Hauer MH, Seeber A, Singh V, Thierry R, Sack R, Amitai A, et al. Histone degradation in response to DNA damage enhances chromatin dynamics and recombination rates. Nat Struct Mol Biol. 2017 Feb;24(2):99-107.

18. Jost $D$, Vaillant $C$. Epigenomics in 3D: importance of long-range spreading and specific interactions in epigenomic maintenance. Nucleic Acids Res. 2018 Mar 16;46(5):225264.

19. Nozaki T, Imai R, Tanbo M, Nagashima R, Tamura S, Tani T, et al. Dynamic Organization of Chromatin Domains Revealed by Super-Resolution Live-Cell Imaging. Mol Cell. 2017 Jul 20;67(2):282-93.e7.

20. Ashwin SS, Nozaki T, Maeshima K, Sasai M. Organization of fast and slow chromatin revealed by single-nucleosome dynamics. Proc Natl Acad Sci U S A. 2019 Oct 1;116(40):19939-44.

21. Germier T, Kocanova S, Walther N, Bancaud A, Shaban HA, Sellou H, et al. Real-Time Imaging of a Single Gene Reveals Transcription-Initiated Local Confinement. Biophys J. 2017 Oct 3;113(7):1383-94.

22. Khanna N, Zhang Y, Lucas JS, Dudko OK, Murre C. Chromosome dynamics near the sol-gel phase transition dictate the timing of remote genomic interactions. Nat Commun. 2019 Jun $24 ; 10(1): 2771$.

23. Shaban HA, Barth R, Recoules L, Bystricky K. Hi-D: nanoscale mapping of nuclear dynamics in single living cells. Genome Biol. 2020 Apr 20;21(1):95.

24. Zidovska A, Weitz DA, Mitchison TJ. Micron-scale coherence in interphase chromatin dynamics. Proc Natl Acad Sci U S A. 2013 Sep 24;110(39):15555-60.

25. Barth R, Bystricky K, Shaban HA. Coupling chromatin structure and dynamics by live super-resolution imaging [Internet]. Cold Spring Harbor Laboratory. 2020 [cited 2020 Nov 2]. p. 777482 . Available from:

https://www.biorxiv.org/content/10.1101/777482v4.abstract

26. Shaban HA, Barth R, Bystricky K. Formation of correlated chromatin domains at nanoscale dynamic resolution during transcription. Nucleic Acids Res. $2018 \mathrm{Jul}$ 27;46(13):e77.

27. Bronshtein I, Kepten E, Kanter I, Berezin S, Lindner M, Redwood AB, et al. Loss of lamin $A$ function increases chromatin dynamics in the nuclear interior. Nat Commun. 2015 Aug 24;6:8044.

28. Gu B, Swigut T, Spencley A, Bauer MR, Chung M, Meyer T, et al. Transcription-coupled changes in nuclear mobility of mammalian cis-regulatory elements. Science. $2018 \mathrm{Mar}$ 2;359(6379):1050-5.

29. Nagashima R, Hibino K, Ashwin SS, Babokhov M, Fujishiro S, Imai R, et al. Single nucleosome imaging reveals loose genome chromatin networks via active RNA 
polymerase II [Internet]. Vol. 218, Journal of Cell Biology. 2019. p. 1511-30. Available from: http://dx.doi.org/10.1083/jcb.201811090

30. Hajjoul H, Mathon J, Ranchon H, Goiffon I, Mozziconacci J, Albert B, et al. Highthroughput chromatin motion tracking in living yeast reveals the flexibility of the fiber throughout the genome. Genome Res. 2013 Nov;23(11):1829-38.

31. Socol M, Wang R, Jost D, Carrivain P, Vaillant C, Le Cam E, et al. Rouse model with transient intramolecular contacts on a timescale of seconds recapitulates folding and fluctuation of yeast chromosomes. Nucleic Acids Res. 2019 Jul 9;47(12):6195-207.

32. Cabal GG, Genovesio A, Rodriguez-Navarro S, Zimmer C, Gadal O, Lesne A, et al. SAGA interacting factors confine sub-diffusion of transcribed genes to the nuclear envelope. Nature. 2006 Jun 8;441(7094):770-3.

33. Amitai A, Seeber A, Gasser SM, Holcman D. Visualization of Chromatin Decompaction and Break Site Extrusion as Predicted by Statistical Polymer Modeling of Single-Locus Trajectories. Cell Rep. 2017 Jan 31;18(5):1200-14.

34. Herbert S, Brion A, Arbona J-M, Lelek M, Veillet A, Lelandais B, et al. Chromatin stiffening underlies enhanced locus mobility after DNA damage in budding yeast. EMBO J. 2017 Sep 1;36(17):2595-608.

35. Eaton JA, Zidovska A. Structural and Dynamical Signatures of Local DNA Damage in Live Cells. Biophys J. 2020 May 5;118(9):2168-80.

36. Lerner J, Gomez-Garcia PA, McCarthy RL, Liu Z, Lakadamyali M, Zaret KS. TwoParameter Mobility Assessments Discriminate Diverse Regulatory Factor Behaviors in Chromatin. Mol Cell. 2020 Aug 20;79(4):677-88.e6.

37. Shaban HA, Seeber A. Monitoring the spatio-temporal organization and dynamics of the genome. Nucleic Acids Res. 2020 Mar 3;48(7):3423-34.

38. Di Stefano M, Paulsen J, Jost D, Marti-Renom MA. 4D nucleome modeling. Curr Opin Genet Dev. 2021 Apr;67:25-32.

39. Doi M, Edwards SF, Edwards SF. The Theory of Polymer Dynamics. Oxford University Press; 1988. $391 \mathrm{p}$.

40. Shi G, Liu L, Hyeon C, Thirumalai D. Interphase Human Chromosome Exhibits Out of Equilibrium Glassy Dynamics [Internet]. Available from: http://dx.doi.org/10.1101/193375

41. Pierro MD, Di Pierro M, Potoyan DA, Wolynes PG, Onuchic JN. Anomalous diffusion, spatial coherence, and viscoelasticity from the energy landscape of human chromosomes [Internet]. Vol. 115, Proceedings of the National Academy of Sciences. 2018. p. 7753-8. Available from: http://dx.doi.org/10.1073/pnas.1806297115

42. Chaki S, Chakrabarti R. Enhanced diffusion, swelling, and slow reconfiguration of a single chain in non-Gaussian active bath. J Chem Phys. 2019 Mar 7;150(9):094902.

43. Foglino M, Locatelli E, Brackley CA, Michieletto D, Likos CN, Marenduzzo D. Nonequilibrium effects of molecular motors on polymers. Soft Matter. $2019 \mathrm{Jul}$ 24;15(29):5995-6005.

44. Liu L, Shi G, Thirumalai D, Hyeon C. Chain organization of human interphase chromosome determines the spatiotemporal dynamics of chromatin loci. PLoS Comput Biol. 2018 Dec;14(12):e1006617. 
45. Ghosh SK, Jost D. How epigenome drives chromatin folding and dynamics, insights from efficient coarse-grained models of chromosomes [Internet]. Vol. 14, PLOS Computational Biology. 2018. p. e1006159. Available from: http://dx.doi.org/10.1371/journal.pcbi.1006159

46. Shukron O, Holcman D. Transient chromatin properties revealed by polymer models and stochastic simulations constructed from Chromosomal Capture data. PLoS Comput Biol. 2017 Apr;13(4):e1005469.

47. Shukron O, Piras V, Noordermeer D, Holcman D. Statistics of chromatin organization during cell differentiation revealed by heterogeneous cross-linked polymers. Nat Commun. 2019 Jun 14;10(1):2626.

48. Strickfaden $\mathrm{H}$. Reflections on the organization and the physical state of chromatin in eukaryotic cells. Genome. 2021 Apr;64(4):311-25.

49. Maeshima K, Ide S, Hibino K, Sasai M. Liquid-like behavior of chromatin. Curr Opin Genet Dev. 2016 Apr;37:36-45.

50. Ashwin SS, Maeshima K, Sasai M. Heterogeneous fluid-like movements of chromatin and their implications to transcription. Biophys Rev. 2020 Apr;12(2):461-8.

51. Strickfaden H, Tolsma TO, Sharma A, Underhill DA, Hansen JC, Hendzel MJ. Condensed Chromatin Behaves like a Solid on the Mesoscale In Vitro and in Living Cells. Cell. 2020 Dec 23;183(7):1772-84.e13.

52. Rao SSP, Huntley MH, Durand NC, Stamenova EK, Bochkov ID, Robinson JT, et al. A $3 \mathrm{D}$ map of the human genome at kilobase resolution reveals principles of chromatin looping. Cell. 2014 Dec 18;159(7):1665-80.

53. Dowen JM, Fan ZP, Hnisz D, Ren G, Abraham BJ, Zhang LN, et al. Control of cell identity genes occurs in insulated neighborhoods in mammalian chromosomes. Cell. 2014 Oct 9;159(2):374-87.

54. Cattoni DI, Cardozo Gizzi AM, Georgieva M, Di Stefano M, Valeri A, Chamousset D, et al. Single-cell absolute contact probability detection reveals chromosomes are organized by multiple low-frequency yet specific interactions [Internet]. Vol. 8, Nature Communications. 2017. Available from: http://dx.doi.org/10.1038/s41467-017-01962-x

55. Lesage A, Dahirel V, Victor J-M, Barbi M. Polymer coil-globule phase transition is a universal folding principle of Drosophila epigenetic domains. Epigenetics Chromatin. 2019 May 13;12(1):28.

56. Finn EH, Pegoraro G, Brandão HB, Valton A-L, Oomen ME, Dekker J, et al. Extensive Heterogeneity and Intrinsic Variation in Spatial Genome Organization. Cell. 2019 Mar 7;176(6):1502-15.e10.

57. Tamm MV, Nazarov LI, Gavrilov AA, Chertovich AV. Anomalous diffusion in fractal globules. Phys Rev Lett. 2015 May 1;114(17):178102.

58. Grest GS, Kremer K. Molecular dynamics simulation for polymers in the presence of a heat bath. Phys Rev A Gen Phys. 1986 May;33(5):3628-31.

59. Chandler D. Introduction to Modern Statistical Mechanics. Oxford University Press, USA; 1987. 274 p.

60. Zidovska A. The self-stirred genome: large-scale chromatin dynamics, its biophysical 
origins and implications. Curr Opin Genet Dev. 2020 Apr;61:83-90.

61. Saintillan D, Shelley MJ, Zidovska A. Extensile motor activity drives coherent motions in a model of interphase chromatin. Proc Natl Acad Sci U S A. 2018 Nov 6;115(45):11442-7.

62. Liu K, Patteson AE, Banigan EJ, Schwarz JM. Dynamic nuclear structure emerges from chromatin crosslinks and motors [Internet]. Available from:

http://dx.doi.org/10.1101/2020.08.22.262758

63. Fudenberg G, Abdennur N, Imakaev M, Goloborodko A, Mirny LA. Emerging Evidence of Chromosome Folding by Loop Extrusion. Cold Spring Harb Symp Quant Biol. 2017;82:45-55.

64. Ghosh SK, Jost D. Genome organization via loop extrusion, insights from polymer physics models. Brief Funct Genomics. 2020 Mar 23;19(2):119-27.

65. Fudenberg G, Imakaev M, Lu C, Goloborodko A, Abdennur N, Mirny LA. Formation of Chromosomal Domains by Loop Extrusion. Cell Rep. 2016 May 31;15(9):2038-49.

66. Sanborn AL, Rao SSP, Huang S-C, Durand NC, Huntley MH, Jewett Al, et al. Chromatin extrusion explains key features of loop and domain formation in wild-type and engineered genomes. Proc Natl Acad Sci U S A. 2015 Nov 24;112(47):E6456-65.

67. Kakui Y, Barrington C, Barry DJ, Gerguri T, Fu X, Bates PA, et al. Fission yeast condensin contributes to interphase chromatin organization and prevents transcriptioncoupled DNA damage. Genome Biol. 2020 Nov 5;21(1):272.

68. Nuebler J, Fudenberg G, Imakaev M, Abdennur N, Mirny LA. Chromatin organization by an interplay of loop extrusion and compartmental segregation. Proc Natl Acad Sci U S A. 2018 Jul 17;115(29):E6697-706.

69. Strom AR, Emelyanov AV, Mir M, Fyodorov DV, Darzacq X, Karpen GH. Phase separation drives heterochromatin domain formation. Nature. $2017 \mathrm{Jul}$ 13;547(7662):241-5.

70. Poonperm R, Hiratani I. Formation of a multi-layered 3-dimensional structure of the heterochromatin compartment during early mammalian development [Internet]. Vol. 63, Development, Growth \& Differentiation. 2021. p. 5-17. Available from: http://dx.doi.org/10.1111/dgd.12709

71. Zhou $Y$, Wang $P$, Tian F, Gao G, Huang L, Wei W, et al. Painting a specific chromosome with CRISPR/Cas9 for live-cell imaging. Cell Res. 2017 Feb;27(2):298301.

72. Alexander JM, Guan J, Li B, Maliskova L, Song M, Shen Y, et al. Live-cell imaging reveals enhancer-dependent Sox2 transcription in the absence of enhancer proximity [Internet]. Vol. 8, eLife. 2019. Available from: http://dx.doi.org/10.7554/elife.41769

73. Brandão HB, Gabriele M, Hansen AS. Tracking and interpreting long-range chromatin interactions with super-resolution live-cell imaging. Curr Opin Cell Biol. 2020 Dec 9;70:18-26.

74. Serra F, Baù D, Goodstadt M, Castillo D, Filion GJ, Marti-Renom MA. Automatic analysis and 3D-modelling of $\mathrm{Hi}-\mathrm{C}$ data using TADbit reveals structural features of the fly chromatin colors. PLoS Comput Biol. 2017 Jul;13(7):e1005665. 
bioRxiv preprint doi: https://doi org/10.1101/2021.05.10.443375. this version posted May 10 2021. The copyriaht holder for this preprint (which was not certified by peer review) is the author/funder, who has granted bioRxiv a license to display the preprint in perpetuity. It is made available under aCC-BY 4.0 International license.

75. Haddad N, Vaillant C, Jost D. IC-Finder: inferring robustly the hierarchical organization of chromatin folding. Nucleic Acids Res. 2017 Jun 2;45(10):e81.

76. Wolff J, Backofen R, Grüning B. Loop detection using Hi-C data with HiCExplorer [Internet]. Cold Spring Harbor Laboratory. 2020 [cited 2021 Jan 8]. p.

2020.03.05.979096. Available from:

https://www.biorxiv.org/content/10.1101/2020.03.05.979096v1.abstract

77. Bonev B, Mendelson Cohen N, Szabo Q, Fritsch L, Papadopoulos GL, Lubling Y, et al. Multiscale 3D Genome Rewiring during Mouse Neural Development. Cell. 2017 Oct 19;171(3):557-72.e24.

78. Rowley MJ, Lyu X, Rana V, Ando-Kuri M, Karns R, Bosco G, et al. Condensin II Counteracts Cohesin and RNA Polymerase II in the Establishment of 3D Chromatin Organization. Cell Rep. 2019 Mar 12;26(11):2890-903.e3.

79. Arbona J-M, Herbert S, Fabre E, Zimmer C. Inferring the physical properties of yeast chromatin through Bayesian analysis of whole nucleus simulations. Genome Biol. 2017 May 3;18(1):81.

80. Milo R, Jorgensen P, Moran U, Weber G, Springer M. BioNumbers-the database of key numbers in molecular and cell biology. Nucleic Acids Res. 2009 Oct 23;38(suppl_1):D750-3.

81. Olarte-Plata JD, Haddad N, Vaillant C, Jost D. The folding landscape of the epigenome [Internet]. Vol. 13, Physical Biology. 2016. p. 026001. Available from:

http://dx.doi.org/10.1088/1478-3975/13/2/026001

82. Sanz E, Marenduzzo D. Dynamic Monte Carlo versus Brownian dynamics: A comparison for self-diffusion and crystallization in colloidal fluids. J Chem Phys. 2010 May 21;132(19):194102.

83. Bal KM, Neyts EC. On the time scale associated with Monte Carlo simulations. J Chem Phys. 2014 Nov 28;141(20):204104.

84. Vaillant $C$, Jost $D$. Modeling the Functional Coupling between 3D Chromatin Organization and Epigenome [Internet]. Modeling the 3D Conformation of Genomes. 2019. p. 21-56. Available from: http://dx.doi.org/10.1201/9781315144009-2 